\title{
Amplification in the Evaluation of Emotional Expressions Over Time
}

\section{A PREPRINT VERSION OF A PAPER PUBLISHED IN NATURE HUMAN BEHAVIOR}

\author{
Amit Goldenberg ${ }^{1^{*}}$ \\ Jonas Schöne $2^{2^{*}}$ \\ Zi Huang ${ }^{1}$ \\ Timothy D. Sweeny ${ }^{3}$ \\ Desmond C. Ong ${ }^{4,5}$ \\ Tim F. Brady ${ }^{7}$ \\ Maria M. Robinson ${ }^{7}$ \\ David Levari ${ }^{1}$ \\ Jamil Zaki ${ }^{6}$ \\ James J. Gross ${ }^{6}$
}

"Equal Contribution

${ }^{1}$ Harvard University, Harvard Business School

${ }^{2}$ University of Oxford, Department of Experimental Psychology

${ }^{3}$ University of Denver, Department of Psychology

${ }^{4}$ National University of Singapore, Department of Information Systems and Analytics

${ }^{5}$ Agency for Science, Technology and Research, Singapore, Institute of High Performance Computing

${ }^{6}$ Stanford University, Department of Psychology

${ }^{7}$ University of California, San-Diego 
Data generated during the studies 1-5 is available in a public repository $\underline{\mathrm{https}}$ ///osf.io/ $\mathrm{krgcv} /$

Social interactions are dynamic, and unfold over time. To make sense of social interactions, people must aggregate sequential information into summary, global evaluations. But how do people do this? To address this question, we conducted 9 studies $(\mathrm{N}=1,583)$, using a diverse set of stimuli. Our focus was a central aspect of social interaction, namely the evaluation of others' emotional responses. Results suggest that when aggregating sequences of images and videos expressing varying degrees of emotions, perceivers overestimate the sequence's average emotional intensity. This tendency for overestimation, which we term the sequential amplification effect, is driven by stronger memory of more emotional expressions. A computational model further supports this account, and shows that amplification cannot be driven merely by nonlinear perception. The current paper is the first to demonstrate amplification in social cognition of sequential information, which is especially important given the prevalence of such information in many social interactions. 


\section{Amplification in the Evaluation of Multiple Emotional Expressions Over Time}

Imagine a friend telling you a story about a situation that made them very angry, or a potential colleague describing an exciting new idea. As the two narratives unfold, various degrees of emotions are expressed over time. These expressions are then summarized into a more general understanding of the person's degree of anger or excitement, a process often termed ensemble coding (Alvarez, 2011; Haberman et al., 2009; Whitney \& Yamanashi Leib, 2018). Exposure to multiple emotional expressions is much more common than observing a single isolated emotional response. However, the vast majority of emotion research has focused on people's ability to evaluate single emotional representations, leaving the question of how people aggregate these sequential emotional representations under-researched.

This gap in our understanding is problematic because there is reason to believe that the aggregation of sequential emotion expressions of differing intensity might be dominated by the stronger emotional responses. This is because stronger emotional expressions are better remembered than weaker expressions (Jackson et al., 2009), which suggests that when an emotional sequence is aggregated, perceivers should overweight stronger expressions and hence, overestimate the sequence's average emotional intensity. The goal of the current set of studies is to evaluate this prediction and assesses its driving mechanisms.

\section{Perceiving Others' Emotions}

Emotional processing is a high priority in person perception. People attend and respond to faces expressing stronger emotional intensity faster (Eimer \& Holmes, 2007; Schirmer \& Adolphs, 2017), and also seem to find it more difficult to detach their attention from more emotional faces (Goldenberg et al., 2020). Increased attention to emotional faces is also associated with better memory of those faces. Both visual working memory (VWM, Jackson et al., 2008, 2009; Lee \& Cho, 2019; Sessa et al., 2011) and long term memory (LTM, Gallegos \& Tranel, 2005; Kaufmann \& Schweinberger, 2004) are enhanced when participants are asked to recall previously shown faces that expressed emotions (versus neutral faces). Differences in the quality of memory are also seen as a function of whether the emotion expressed was positive or negative, but results vary across studies. While some studies suggest that people remember faces with negative expressions better than those with positive expressions (Jackson et al., 2008, 2014; Sessa et al., 2011), others find that memory for 
positive faces is enhanced (D’Argembeau \& Van der Linden, 2007; Kaufmann \& Schweinberger, 2004). A recent study by Lee and Cho (2019) suggests that VWM is enhanced for negative faces, while LTM is enhanced for positive ones.

How might the priority given to more versus less emotional expressions affect people's perception of multiple emotion expressions that occur sequentially? One clue is provided by the observation that people often are likely to remember only a subset of items within a sequence (Tong et al., 2019). For example, working visual memory allows the recollection of four items, more or less (Luck \& Vogel, 1997; Wilken \& Ma, 2004). Given the primacy that emotional faces have in memory, salient expressions may be more likely to be preferentially remembered in long sequences. When asked to evaluate the average of a certain sequence, people might then evaluate the overall emotional response as more intense than it actually is (Fiedler, 2000). Furthermore, as longer sequences are more likely to contain stronger emotional responses, there are more opportunities to remember more emotional expressions. Therefore, simply as a matter of sampling, amplification is likely to be stronger for longer sequences. Finally, given that visual working memory is enhanced for negative faces, it is possible that when asked to average a sequence of emotions immediately after they occurred, amplification would be larger for negative emotions, compared to positive. To address this possibility, it's useful to consider findings regarding ensemble coding.

\section{Ensemble Coding of Sequences}

Ensemble coding involves extracting summary statistics, typically the mean, but sometimes measures of variability, from multiple stimuli quickly and accurately (Alvarez, 2011; Haberman \& Whitney, 2007; Whitney \& Yamanashi Leib, 2018). While a large portion of ensemble coding research has focused on multiple stimuli appearing concurrently, there has been an increasing interest in ensemble coding of sequential stimuli (Albrecht \& Scholl, 2010; Haberman et al., 2009; Juni et al., 2012; Oriet \& Brand, 2013; Piazza et al., 2013). As much of our world, and especially our social world, unfolds over time, learning about how people summarize and make sense of sequential information is of upmost importance.

There are only a handful of papers that examines ensemble coding of emotional sequences, and two general conclusions can be taken from these works. First, people are generally accurate in evaluating the average emotion expressed in a sequence of faces (Haberman et al., 2009). Second, when evaluating emotional sequences, people tend to overweight faces that are more recent (recency 
bias, Hubert-Wallander \& Boynton, 2015). This recency bias effect is due to the fact that memory tends to decay over time, thus providing priority to more recent faces (Huang \& Sekuler, 2010).

While people may be generally accurate in evaluating sequences of faces (Haberman et al., 2009), the fact that memory is enhanced for more emotional faces (D’Argembeau \& Van der Linden, 2007; Jackson et al., 2014; Kaufmann \& Schweinberger, 2004; Sessa et al., 2011) would predict that people's evaluations may be skewed towards evaluating averages of emotional sequences as more emotional than they actually are. Some findings in ensemble coding research provide indirect support for this hypothesis on non-emotional stimuli. For example, in two studies that examined people's ability to evaluate the average size of circles appearing in sequence, people overweighted larger circles in estimating the mean size (Albrecht \& Scholl, 2010; Hubert-Wallander \& Boynton, 2015). One alternate explanation is that larger circles take up more of one's visual field. There are also some indirect indications of amplification in the evaluation of sequences of money (Putnam-Farr \& Morewedge, 2020; Tsetsos et al., 2012), which can be interpreted as congruent under the assumption that larger sums of money are more salient in memory than smaller sums. Furthermore, recent findings from ensemble coding of non-sequences suggest that people may overestimate the average size of arrays of circles (Kanaya et al., 2018) and even of the emotional intensity of crowds (Goldenberg et al., 2020).

Additional indirect support for the idea that people may be biased in estimating sequences of emotion comes from the peak-end rule (Fredrickson, 2000; Kahneman et al., 1993; Redelmeier \& Kahneman, 1996). According to the peak-end rule, people evaluate subjective experiences by averaging the peak and the end of the experience (Cojuharenco \& Ryvkin, 2008; Kahneman et al., 1993). While the peak-end rule is congruent with some of the current hypotheses, there are also clear differences that warrant additional research. First, the peak-end rule only has been examined by evaluating people's subjective experience, rather than social perceptions. Second, the peak-end rule focuses only on the peak and the end of a sequence, while we wish to argue the memory promotes more salient expressions in general, not only the peak, which should lead to different predictions in degree of amplification (see Supplementary Materials for direct comparisons). Finally, Kahneman and colleagues argue that the length of the sequence should not affect its affective evaluation (duration neglect, Redelmeier \& Kahneman, 1996), while we argue that length matters.

\section{The Present Research}


We conducted a set of 9 studies with the goal of identifying the occurrence of amplification in the evaluation of emotional sequences and its driving mechanisms. The first set of studies (Studies 1ad) was designed to detect the existence of amplification in the evaluation of emotional sequences, addressing potential challenges to the main finding in each study. These studies had three main preregistered hypotheses (https://osf.io/ag8nv). Our first hypothesis was that participants would estimate the average emotion expressed in a sequence of emotions as more intense than it actually is (amplification effect). Our second hypothesis was that amplification would be stronger for longer sequences. Our third hypothesis focused on the difference between positive and negative sequences. While we expected amplification to be stronger for negative compared to positive emotions, based on different findings in the memory literature, we also expected that the difference between negative and positive emotions to be relatively small and inconsistent (see Goldenberg et al., 2020).

Having found evidence for the three hypotheses, the second set of studies (Studies 2-6) was designed to replicate the findings from Studies 1a-d, and to test the possibility that enhanced memory to emotional faces was driving the sequential amplification effect. In Study 2, we validated two assumptions regarding the connection between memory and perception of emotional sequences. The first was that recent faces in the sequence are more salient in memory. The second was that salient items in the sequence, in our case stronger emotions, are also more likely to be remembered. In Study 3, we took advantage of the fact that recent faces were more likely to be remembered and examined how recency of high and low emotional intensity affected amplification. In Study 4, we manipulated the salience of either high or low intensity faces, using red frames around these faces, in an attempt to impact memory and therefore amplification. In Study 5, we compared three potential computational models of ensemble coding to made sure that amplification was not driven merely by non-linearity in emotion perception. These studies provide additional evidence for the idea that memory for some items in the sequence is one key driver of amplification. Finally, in Study 6, we addressed limitations in external validity and examined the occurrence of amplification in the evaluations of natural videos depicting emotional stories using existing data from the Stanford Emotional Narratives Dataset (Ong et al., 2019).

\section{Studies 1a-1d: Establishing Amplification in the Evaluation of Sequence of Faces}

The goal of Studies 1a-1d was to test the three hypotheses described above (https://osf.io/ag8nv). Study 1a was a first attempt to capture the amplification effect. Study $1 \mathrm{~b}$ 
replicated the effect using a different stimulus set. Study $1 \mathrm{c}$ and $1 \mathrm{~d}$ examined whether amplification persisted despite changes to the measurement scale (1c) and its starting point (1d).

\section{Methods}

Participants. Our pre-registered power analysis of Study 1a was based in previous findings that showed amplification in crowd estimation (Goldenberg et al., 2019) in which we concluded that 30 participants completed 50 trial would be satisfying to achieve a power of $100 \%$. However, this preregistration was designed for a lab study. Given that we decided to run all of our studies online, we decided to increase our sample size to 100 participants per study, which was our sample size in all sequential amplification studies throughout. Of the 100 participants that completed the task, we removed participants whose average estimation was below 10 or higher than 40 (see further explanation below), which could only occur if ratings were conducted in order to finish the task quickly without any regard to the averages. These elimination criteria were not preregistered in this study due to an oversight, which was corrected in all other pre-registrations and is similar in all studies. Furthermore, including these participants did not change the main effects. Our starting sample was 100 participants in each of the four studies, after removing participants based on the above criteria or for missing demographic data, our final sample was: Study 1a: N=93; men: 52, women: 41; age: $\mathrm{M}=28.23$, SD = 9.54, Study 1b: $\mathrm{N}=94$; men: 35, women: 55 other/did not specify = 4; age: $M=33.60, S D=11.43$, Study 1c: $N=98$; men: 35, women: 62 , other/did not specify= 1 ; age: $M=$ 25.78, SD = 9.08, Study 1d: N=92; men: 31, women: 66 , other/did not specify=1; age: $M=24.67, \mathrm{SD}=$ 6.42 (see breakdown of participants' race in Supplementary materials). All of our participants were recruited through Prolific and received \$2.30 for their participation.

Stimuli. To create the stimuli set for our task, we created face morphs from two face sets. The first set was developed for a recent investigation of ensemble face perception (Elias et al., 2017) and was based on four exemplar faces of men from the NimStim face set (Tottenham et al., 2009). For each identity in the set, and for two type of emotions for each identity: happiness and anger, the original authors created a morphed scale of o\% - completely neutral to 100\%-completely emotional in increments of $2 \%$ (1-50 scale, see Figure 1). This meant that each identity had a two sets of 50 faces expressing emotions from neutral-to-happy and neutral-to-angry. These sets were piloted to make sure that participants evaluation of a single face was accurate. In order to increase the diversity of our morphed faces we created a second morphed set, using eight exemplar faces ( 4 women, 4 men) based 
on the Radboud face sample (Langner et al., 2010). We created the second sample both to increase the validity of the task, as well as to increase the gender diversity of the target faces, which were all men in the first morph set. The procedure of creating the second morphed set was as similar as possible to the first one, creating two sets neutral-to-happy and neutral-to-angry sequence for each face using the morphing software Fantamorph. We conducted a pre-test to confirm that the evaluation of a single face was accurate, finding no amplification effect for a single face evaluation (see Supplementary Materials).

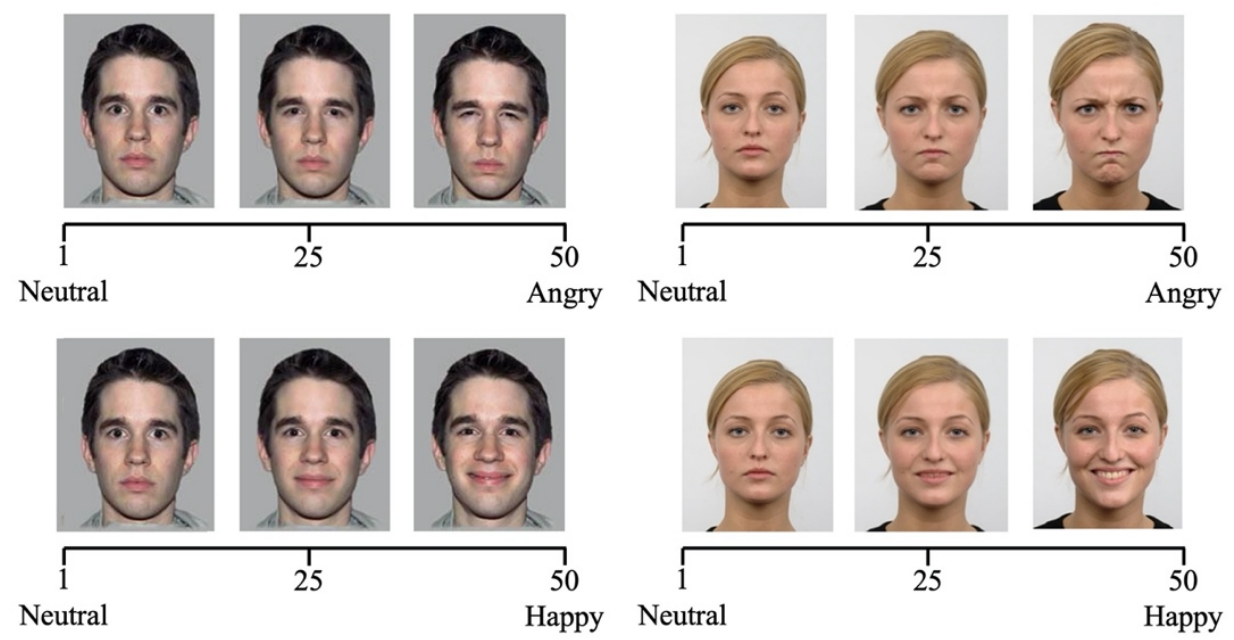

Figure 1. Two samples (NimStim - left, and Radboud - right) of three faces from the neutral-to-angry scale (top) and from the neutral-to-happy scale (bottom) that were used in the studies. Values of 25 and 50 correspond to $50 \%$ and $100 \%$ intensities in our morph range, respectively.

Procedure. Ethics committee approval was obtained prior to the collection of data. In each trial, participants first saw a sequence of 1-12 faces with the same identity expressing different intensities of emotion from either a neutral-to-angry (anger condition) or a neutral-to-happy (happiness condition) continua (Figure 1). Each face in the sequence was presented in the middle of the screen for 100oms on a grey background and was randomly taken from a 1-50 morph, 1 corresponding to a neutral face, and 50 to the most-angry/most-happy face (Figure 2). Between each face, participants saw a fixation cross for a duration between 400-60oms (randomly determined on each trial). The sequence's average expressive intensity was therefore normally distributed around 25.5 which was the middle of the scale, with varied degrees of variance. The valence and the number 
of faces presented in each sequence were also chosen randomly in each trial. We did not mix the happy and angry faces in the same sequence for two reasons. First, doing so could undermine our ability to detect an amplification effect: if participants fixated on one extremely negative and one extremely positive face, then on average, their estimate of the sequence could appear to be relatively accurate despite the fact that they were biased by emotional intensity in their sampling of faces. Second, the most-happy and most-angry faces may not be considered equal in intensity, thus making the average between the two different from zero.

A

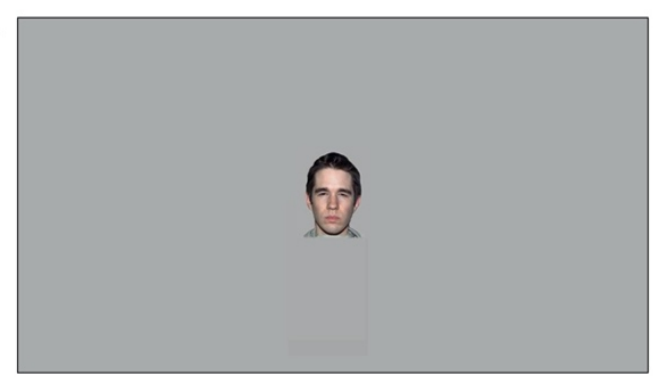

1 seconds

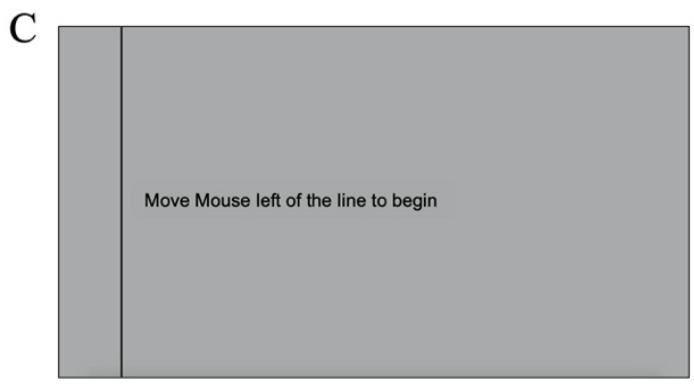

B

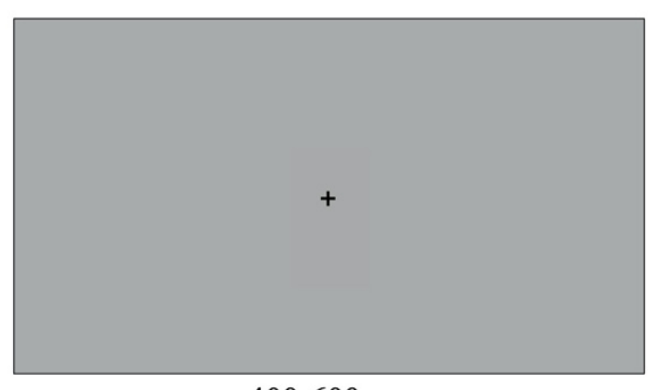

$400-600 \mathrm{~ms}$

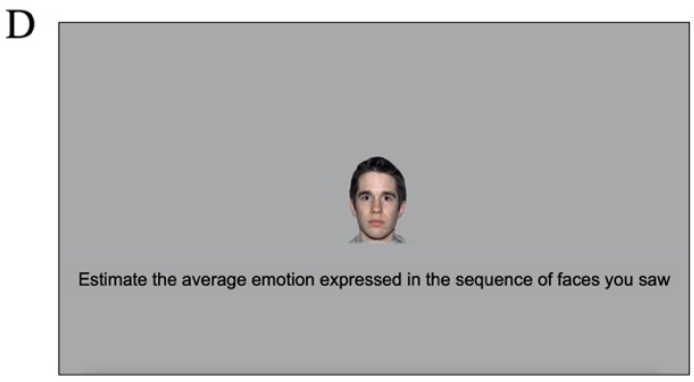

Figure 2. The task used in Studies 1a-d. Participants saw a sequence of 1-12 faces expressing either different degrees of anger or happiness that appeared on the screen for one second (A represents one face in the sequence). Between each face participants saw a fixation cross for 400-600 ms (B). Participants were then asked to move the mouse to the left of the line to begin the evaluation stage $(\mathrm{C})$. They were then asked to evaluate the average emotion expressed by these faces by adjusting the intensity of a single morphed face (1-50, D).

Following the sequence of faces, participants were asked to evaluate the average emotion expressed in the sequence. To start the measurement phase, participants were asked to move their cursor beyond a vertical line which was located on the left (Studies 1a, 1b) or the right side of the screen (Studies 1c, 1d, see Figure 1C). Once the pointer crossed the vertical line, a single face bearing a neutral expression was presented on the screen (Figure 1D). The identity of the face in the scale 
matched that of the faces in the previous sequence. Participants were then asked to move the pointer away from the starting point to modify the face from neutral to emotional. Participants had as much time as they needed to estimate the mean intensity of the emotional sequence. We modified the location and starting point of the scale in Studies 1a-1d. In Studies 1a and 1b, the initial face in the scale was anchored on a neutral expression starting on the left side of the screen: 1 on the scale from 1 - 50. This was done because previous research indicated that the initial location of the scale in ensemble coding tasks led to an anchoring effect, such that estimations were closer on average to that of the initial location (see for example Oriet \& Brand, 2013). In Study 1c, the scale also started from neutral, but the direction of the scale was reversed, from right to left. This was done to make sure that the effect is not amplified due to the direction of the scale. Finally, in Study 1d, the scale was initiated on the left side of the screen but the starting point of the scale was the most emotional face. This was done to eliminate the possibility that starting from neutral led participants to "overshoot" in their estimation of the average.

After completing the main task, participants filled out a short survey which included the social interaction anxiety scale (SIAS, Mattick \& Clarke, 1998), a PANAS scale (Watson et al., 1988), a BigFive personality scale (Gosling et al., 2003), a three-item loneliness scale (Hughes et al., 2004), and demographic questions including age, gender, race and education level. These scales were administered for exploratory purposes (i.e., in order to examine potential moderators), so the corresponding results are reported in the Supplementary Materials.

\section{Results}

We tested three hypotheses in our main analysis. To measure general amplification in estimation of the face sets (H1), we conducted a mixed model analysis of repeated measures, comparing the actual mean emotion expressed in each set with participants' estimated mean emotion. As each participant was exposed to four face identities, we added random intercepts of participant id and face identity id (a model with a slope of trial number failed to converge for most analyses). Supporting the first hypothesis, estimated mean emotion was higher than the actual mean emotion in all of the studies (see Table 1). When the scale started with high intensity rather than neutral the amplification effect was more than double in size, supporting the claim that starting the scale with a neutral rating is a conservative estimate of the effect. 
The second and third hypotheses were tested with a single model. For our dependent variable, we created a difference score between participants' estimation of the sequence average and the actual average, and we used both sequence length $\left(\mathrm{H}_{2}\right)$ and the valence $\left(\mathrm{H}_{3}\right)$ as predictors. In an initial version of the model, we included an interaction between length and valence, but this did not improve the model fit. As in the previous analysis, we added a by-participant random intercept. Our results supported our second hypothesis; increase in the number of faces in the sequence led to an increase in amplification in all of the studies. Further analysis conducted on sequence length (reported in detail in SM), indicated that amplification was evident only in sequences larger than 4-6 faces, depending on the study, which is congruent with research on working visual memory. Finally, we found increased amplification for negative sequences compared to positive sequences in 3 out of 4 of our studies, and as expected, even in the studies in which this effect was found it was relatively weak.

Table 1. Summary of results of Studies 1a-d divided by the three hypotheses. The first hypothesis was that participants would tend to evaluate the sequence mean as more intense than it actually was. The second hypothesis was that amplification in the evaluation of the sequences will increase with sequence length. The third hypothesis was that amplification would be stronger in negative sequences.

\begin{tabular}{|c|c|c|c|c|}
\hline Hypothesis & Study & b [ci], (se) & $t(d f)$ & $\mathbf{p}$ \\
\hline \multirow{4}{*}{$\begin{array}{l}\text { H1: General } \\
\text { amplification } \\
\text { (positive numbers } \\
\text { indicate } \\
\text { amplification) }\end{array}$} & 1a: Establishing effect & $.75[.41,1.09],(.17)$ & $4 \cdot 35(9,704)$ & $<.001^{* \star *}$ \\
\hline & $\begin{array}{l}\text { 1b: Replication with a } \\
\text { new morph set }\end{array}$ & $1.40[1.07,1.72],(.16)$ & $8.50(8,964)$ & $<.001^{* *}$ \\
\hline & $\begin{array}{l}\text { 1c: Scale starts on } \\
\text { right side }\end{array}$ & $1.31[.98,1.65],(.17)$ & $7 \cdot 71(10,012)$ & $<.001^{* \star *}$ \\
\hline & $\begin{array}{l}\text { 1d: Scale starts with } \\
\text { strong intensity }\end{array}$ & $3.95[3.60,4.30],(.17)$ & $22.22(9,395)$ & $<.001^{* \star *}$ \\
\hline \multirow{3}{*}{$\begin{array}{l}\text { H2: Amplification } \\
\text { as a function of } \\
\text { sequence length } \\
\text { (positive numbers } \\
\text { indicate increased }\end{array}$} & 1a: Establishing effect & $.33[.25, .40],(.03)$ & $8.91(4,820)$ & $<.001^{\star * \prime}$ \\
\hline & $\begin{array}{l}\text { 1b: Replication with a } \\
\text { new morph }\end{array}$ & $.30[.22, .36],(.03)$ & $8.45(4,428)$ & $<.001^{* * *}$ \\
\hline & $\begin{array}{l}\text { 1c: Scale starts on } \\
\text { right side }\end{array}$ & $.36[.29, .43],(.03)$ & $10.36(4,988)$ & $<.001^{* * *}$ \\
\hline
\end{tabular}


AMPLIFICATION OVER TIME

\begin{tabular}{|c|c|c|c|c|}
\hline $\begin{array}{l}\text { amplification with } \\
\text { sequence length) }\end{array}$ & $\begin{array}{l}\text { 1d: Scale starts with } \\
\text { strong intensity }\end{array}$ & $.19[.12, .26],(.03)$ & $5 \cdot 36(4,965)$ & $<.001^{\star \star *}$ \\
\hline \multirow{4}{*}{$\begin{array}{l}\text { H3: Amplification } \\
\text { as a function of } \\
\text { sequence valence } \\
\text { (positive numbers } \\
\text { indicate that } \\
\text { negative sequences } \\
\text { were stronger than } \\
\text { positive sequences) }\end{array}$} & 1a: Establishing effect & $.56[.232,1.07],(.26)$ & $2.20(4,823)$ & $.02^{*}$ \\
\hline & $\begin{array}{l}\text { 1b: Replication with a } \\
\text { new morph }\end{array}$ & $.91[.43,1.39],(.24)$ & $3 \cdot 74(4,420)$ & $<.001^{* * *}$ \\
\hline & $\begin{array}{l}\text { 1c: Scale starts on } \\
\text { right side }\end{array}$ & $.40[-.06, .88],(.24)$ & $1.68(4,987)$ & .09 \\
\hline & $\begin{array}{l}\text { 1d: Scale starts with } \\
\text { strong intensity }\end{array}$ & $-.77[-1.28,-.27],(.25)$ & $-3.03(4,967)$ & $<.001^{* * *}$ \\
\hline
\end{tabular}

Studies 1a-d provide evidence for the occurrence of amplification in the evaluation of sequences expressing different degrees of emotion (Figure 3). However, these studies do not yet shed light on the mechanisms that may drive such amplification. In the next three studies, we attempt to examine the idea that enhanced memory to salient faces, in this case emotional faces, is driving amplification.

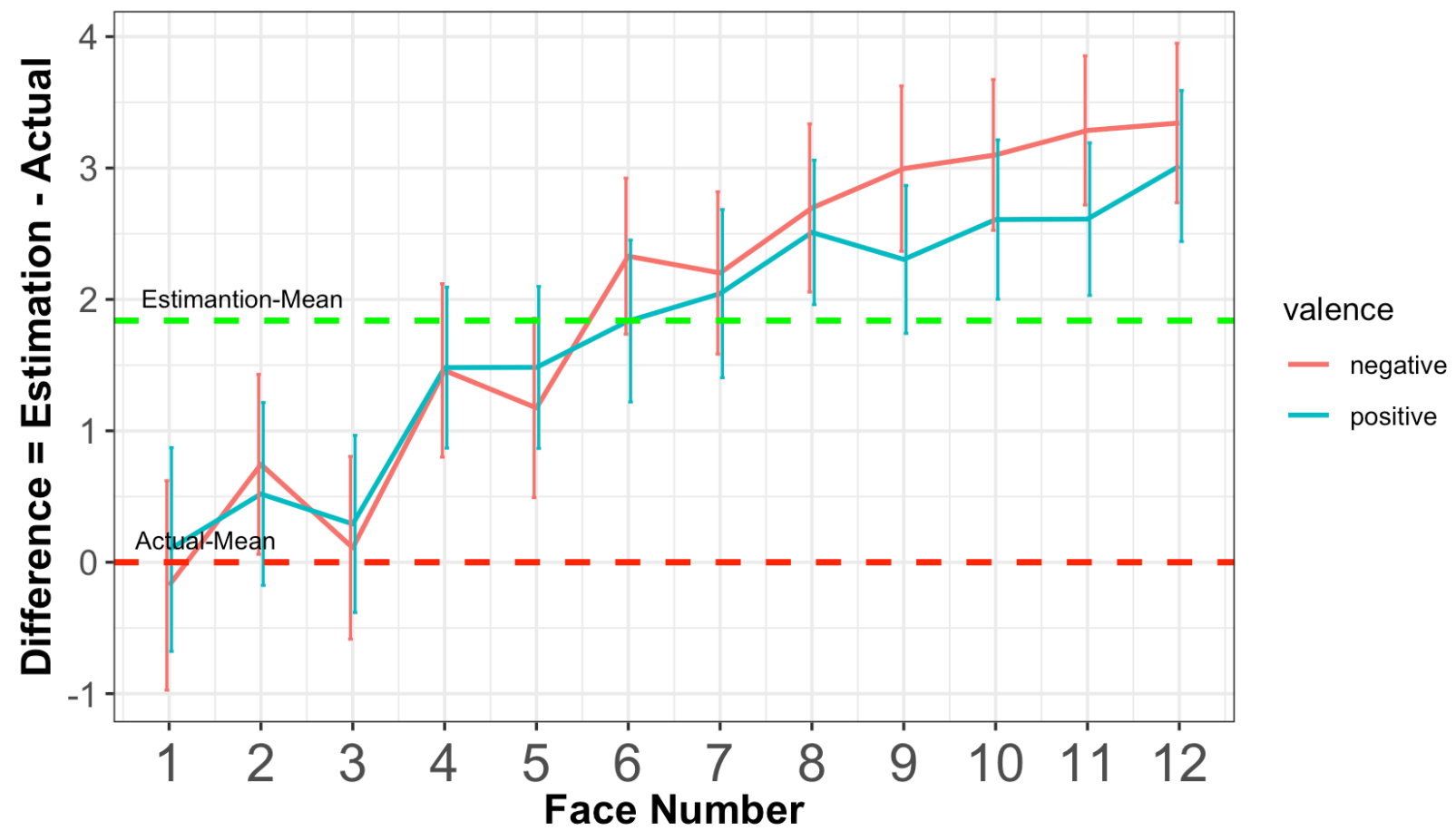

Figure 3. Summary of Studies 1a-1d results. The x-axis represents the number of faces in the sequence. The y-axis represents the difference between participants' estimation of the average 
sequence and the actual average. The green dotted line represents the average amplification across studies. The red dotted line represents the actual face mean.

\section{Study 2: Testing the Impact of Recency and Emotionality on Participants' Evaluation}

The goal of Study 2 was to validate two previously reported findings related to the importance of memory to sequence evaluation, with the hope that they could be used as building blocks for manipulating memory salience in Studies 3 and 4. Study 2 included two blocks. In the first block, participants were asked to evaluate the average emotion expressed in sequences of 8 faces. We hypothesized we'd see amplification in this block (H1) as well as stronger amplification for negative emotions (H2, pre-registration: https://osf.io/j4kqz/). In addition, we kept the size of the sequences constant in order to evaluate the degree to which each face in the sequence predicted participants' estimation of the average, but we did not pre-register a hypothesis for this analysis. In the second block, participants saw sequences of 8 faces and then performed a memory test by choosing between a true-target expression that appeared in the sequence and a false-target expression that did not appear in the sequence. We hypothesized that people would be more likely to succeed in the memory test in the trials in which the true-target face expressed stronger emotion $\left(\mathrm{H}_{3}\right)$. We also examined whether the relative location of the true-target in the sequence predicted memory (not pre-registered). Finally, in an exploratory analysis, we examined whether individual tendency to better remember more intense emotions (measured in block 2) predicted increased amplification (measured in block 1).

\section{Methods}

Participants. We used results from Study 1a to evaluate the required sample size (see full description in the Supplementary Materials). Power analysis suggested that a sample of 100 participants completing 20 trials in each block would provide power of almost 100\%. However, running a study with this sample indicated that amplification is lower when the sequence length remained constant (see full report in SM). We therefore used the findings of this first attempt to generate an updated prediction for sample size which suggested that 150 participants completing 30 trials for the amplification block and 20 trials to the memory block would be suitable to capture an amplification effect with a power of $86 \%$. We therefore repeated data collection and collected a new, larger sample. Based on our pre-registered criteria and similar to all other studies, we removed participants whose average estimation was below 10 or higher than 40 which could only occur if ratings were conducted in order to finish the task quickly without any regard to the averages. Our 
starting sample was 150 participants, but we were left with 136 participants after removing participants based on our pre-registered criteria. Given that 137 participants would put us under power of $80 \%$, we then collected additional 13 participants prior to examining or analyzing these data, bringing us to $\mathrm{N}=150$ (men: 51, women: 98 , 1 Other; age: $\mathrm{M}=38.35$, SD $=12.37$ ). All of our participants were recruited through Prolific and received $\$ 2.30$ for their participation.

Procedure. The task included two separate blocks that were always presented to participants in the same order. The first block was the amplification test block which was 30 trials long. Participants completed an amplification task similar to that of Studies 1a-d with one difference: the length of the sequence was always eight faces. We kept the sequence length equal for two reasons. First, we wanted our amplification trials to be of the same sequence length as the memory trials. Second, we wanted to be able to compare the contribution of the order of each face in the sequence to participants' estimation. The second block, which was 20 trials long, was designed to examine participants' ability to remember certain emotional expressions in the sequence. Similar to the first block, each trial started with a sequence of 8 faces, either neutral-to-negative and neutral-to-positive, with each face randomly drawn from the 1-50 scale. Following each sequence, participants saw two target faces: a true target that appeared in the sequence and a false target that did not appear in the sequence. The false target expressions were chosen in each trial by finding the two faces that appeared in the sequence that had the biggest difference between them and taking the mid-point of that difference. For example, if 5 and 13 had the biggest difference in the sequence, the false target face would be 9. The two target face appeared on the screen right after the final fixation cross of the sequence (400-60o ms). Participants had as much time as they needed to make their choice. Following the task, participants completed a survey similar to previous studies (see SM for full analysis).

\section{Results}

We started our analysis by looking at the first block in which participants were asked to evaluate the average intensity of the sequence. To measure general tendency for amplification, we conducted a mixed model analysis in which we compared participants' estimation of the sequences to the actual average, using by-individual and face-identity random intercepts. Similar to Studies 1a-d and as predicted in Hypothesis 1, results suggested that participants evaluated the sequence as more emotional than it actually was $\left(b=.59[.30,89], S E=.14, t(8,846)=4.02, p<.001, R^{2}=.10\right)$, 
although the effect was smaller than those found in Studies 1a-d. This may be due to the fact that sequence length didn't change which made it easier for participants to improve on the task, which is also evident by the lower SE compared to Studies 1a-d (.14 in this study compared to $.16-.17$ in Studies 1a-d) despite having less trials than those studies. We then compared the amplification of negative and positive sequences. This was done by looking at the difference score between participant's estimation of the average sequence as the dependent variable and using valence as the independent variable. Similar to previous models, the model used participant-id and face-identity as random intercepts. As predicted in Hypothesis 2, results suggested that amplification of negative sequences was significantly stronger than positive sequences $\left(b=1.12[.30,89], S E=.23, t(8,849)=4.72, p<.001, R^{2}=.26\right)$.

After confirming the general effect of amplification, we examined the weight given to each of the faces in the sequence. This was done in a similar way to Hubert and colleagues (Hubert-Wallander \& Boynton, 2015), by using each face number as a separate coefficient in a mixed model regression predicting participants' estimation. We removed the intercept of the model to get a comparison of each coefficient to zero as well as added a by-participant and face-identity as random intercepts, similar to all other analyses. See the left size of Table 2 (Face Number Predicting Estimation) for the size of each coefficient. As expected, and aligned with previous findings, recent faces in the sequence were stronger predictors of participants' estimation of the sequence average, which would suggest that they were more salient in participants' memory.

Table 2. Results for the two analyses that examined the order of the face in the sequence predicting either participants' estimation of the average sequence emotionality (left) or whether participants were able to recall the face in the memory test (right).

\begin{tabular}{|c|c|c|c|c|}
\hline \multirow{2}{*}{$\begin{array}{c}\text { Face } \\
\text { Location }\end{array}$} & \multicolumn{2}{|c|}{$\begin{array}{c}\text { Face Location Predicting } \\
\text { Estimation }\end{array}$} & \multicolumn{2}{c|}{$\begin{array}{c}\text { Face Location Predicting } \\
\text { Correct Memory }\end{array}$} \\
\cline { 2 - 5 } & $\boldsymbol{b}[\mathrm{ci}]$, & $\boldsymbol{t}$ & $\boldsymbol{b}[\mathrm{ci}]$ & $\boldsymbol{z}$ \\
\hline 1 & $.04[.02, .06]$ & $5.80^{* * *}$ & $-.04[-.23, .15]$ & -.44 \\
\hline 2 & $.04[.02, .06]$ & $5.59^{* * *}$ & $-.01[-.20, .17]$ & -.18 \\
\hline 3 & $.05[.03, .06]$ & $6.61^{* * *}$ & $-.11[-.08, .31]$ & -1.16 \\
\hline 4 & $.05[.04, .07]$ & $7.46^{* * *}$ & $-.09[-.30, .11]$ & -.91 \\
\hline 5 & $.07[.05, .08]$ & $9.45^{* * *}$ & $.12[-.07, .32]$ & 1.18 \\
\hline
\end{tabular}


AMPLIFICATION OVER TIME

\begin{tabular}{|c|c|c|c|c|}
\hline 6 & $.07[.05, .08]$ & $8.87^{* * *}$ & $-.03[-.22, .16]$ & -.32 \\
\hline 7 & $.08[.06, .09]$ & $10.48^{* *}$ & $.31[.12, .50]$ & $3.20^{* *}$ \\
\hline 8 & $.011[.09, .012]$ & $14.16^{* * *}$ & $.53[.33, .74]$ & $5.16^{* * *}$ \\
\hline
\end{tabular}

Having established both amplification and recency effects, we then turned to the memory block, examining whether the emotional intensity of the target face predicted the probability of remembering the face (Hypothesis 3). To evaluate this question, we conducted a mixed generalized linear model in which we used the emotional intensity of the true target expression as predictor and whether participants chose this face correctly or not as the dependent variable. We added a covariate to the model of the distance between the false and the true target, as such distance is likely to affect participants ability to remember. We also added a by-individual random intercept and a random intercept of the face identity. As hypothesized, results suggested that the intensity of the face predicted the probability of memory $(b=.04[.37, .47], S E=.002, z=15.23, p<.001$, Figure 4$)$. To make sure that this effect was not driven solely by participants merely choosing the more emotional face in each trial, we reduced the dataset to the cases in which the true-target face was lower in intensity than the false target face and redid the analysis. The effect remained significant within this subset despite cutting the sample size by half $(b=.01[.001, .029], S E=.007, z=2.16, p=.03)$. We did not find a significant main effect for valence on participants' memory as well as an interaction between the intensity of the true-target stimuli and its valence on the probability of memory (see SM). 


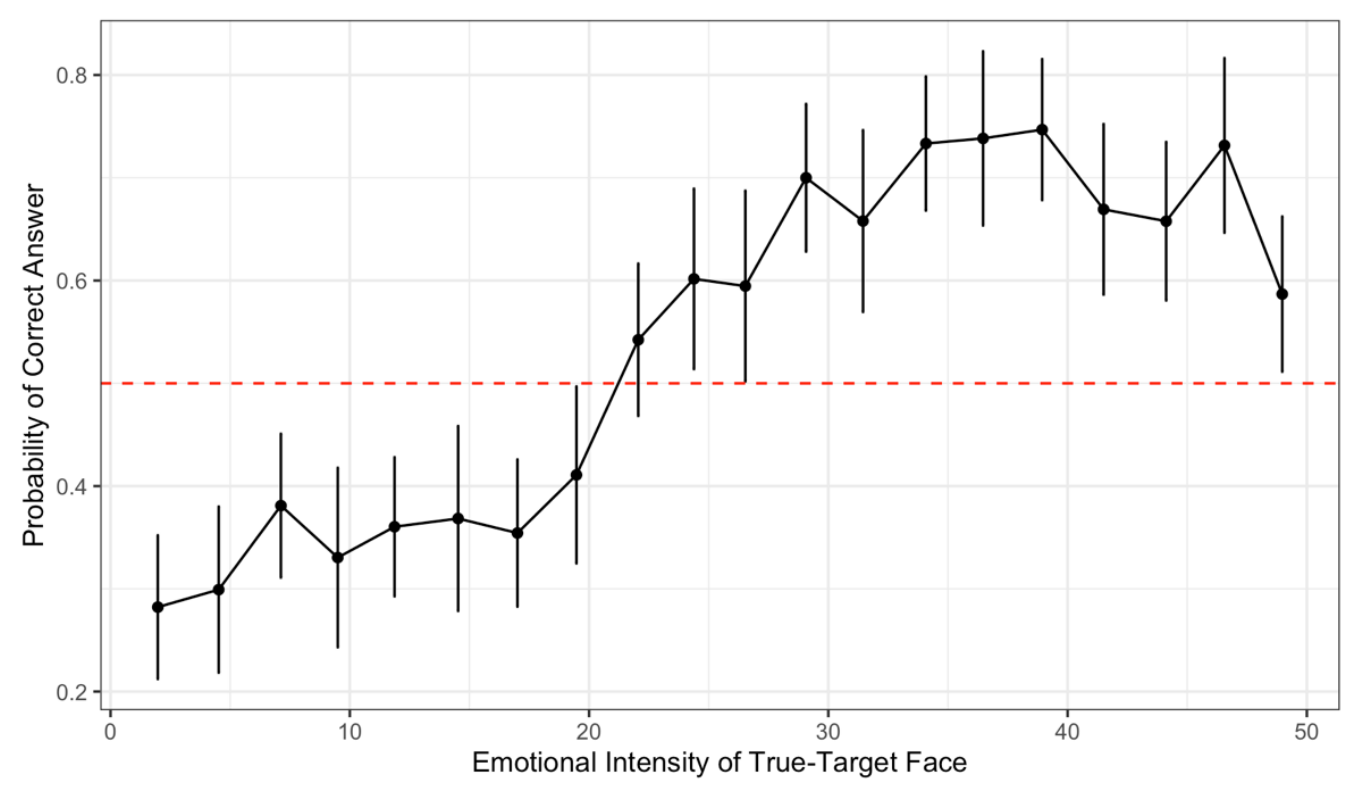

Figure 4. Emotional intensity of the true-target face predicting the probability for correct answer. We aggregated the data into 20 slots in order to provide an estimate of the standard error of the predictions for the sake of visualization. Red dotted line represents chance accuracy.

In addition to testing whether emotional salience predicted memory, we also wanted to replicate the recency findings from the first block by examining whether true-target faces were more likely to be identified if they appeared later in the sequence. To evaluate this question, we created a binary value for each of the 8 faces that participants saw in each trial, zero indicating that a specific face was not the true target face and 1 indicating that it was. For most trials, there was only one value in the sequence that was similar to the true-target face, but in $13.43 \%$ of cases the true target face corresponded to two or more faces in the sequence. We then conducted a mixed model analysis in which we used each of these binary values for each face as the independent values, and whether participants were correct in the memory test as the dependent variable. The model included byindividual and by-face-identity random variables and we removed the intercept of the model to evaluate the importance of each face location, similar to the model that attempted to evaluate the importance of location on amplification. As seen in the right side of Table 2, the order of the truetarget faces in the sequence predicted whether the memory was correct only at the $7^{\text {th }}$ or $8^{\text {th }}$ order. This finding provides some support to the notion of recent items being more salient in memory. In addition, these findings can be seen as providing additional evidence to the memory task was quite difficult. 
One exploratory hypothesis that was proposed in our pre-registration was that individuals' tendency to correctly remember stronger stimuli could potentially predict tendencies for amplification. To examine this possibility, we created a coefficient for each participant of face emotional intensity predicting memory: specifically, we ran a generalized logistic regression for each participant looking at emotional intensity of the true target face as a predictor the probability of memory (face emotional intensity predicting memory). We then took the regression coefficient for each participant, such that a positive coefficient indicated that, for that participant, increased intensity predicted better memory. The intensity-memory coefficients were then used to predict tendency for amplification in the sequence evaluation block. This was done by running a mixed model analysis using the participant-level memory coefficient as a predictor and the difference between the estimated sequence intensity and the actual intensity in each trial. Similar to previous analyses, we added byindividual and by-face-identity random intercepts. Results suggested that the association between the memory-intensity coefficient and participants' tendency for amplification was non-significant ( $b$ $=5.94[.30,89], S E=7.17, t(147)=.83, p=.40)$. This means that individual level tendencies for memory did not predict trial level amplification.

Results of Study 2 point to two important findings regarding the connection between memory and sequence evaluation. The first is that recent items (i.e., faces that appeared toward the end of the sequences) were more salient in participants' memory. This was seen both in the fact that recent items were found to be more strongly associated with participants' estimation, and that they were more likely to be correctly remembered. The second finding was that salience, in this case salience due to emotional intensity, was more likely to be remembered and potentially therefore to impact one's evaluation of the sequence. We hoped to manipulate both order and salience to further examine their effect on amplification in Studies 3 and 4.

\section{Study 3: Manipulating Recency of Strong Intensity Emotions}

The goal of Study 3 was to examine the effect of memory on participants' tendency for amplification by manipulating the intensity of emotions that participants viewed either at the beginning or the end of the sequence. Our pre-registered hypotheses (https://osf.io/sgbzy/) were: that we would see amplification (H1), that amplification would increase with sequence length (H2) and that amplification would be stronger for negative emotions (H3). Finally, we hypothesized that 
amplification would be stronger for trials in which stronger emotions were presented at the end of the sequence, compared to the beginning $\left(\mathrm{H}_{4}\right)$.

\section{Methods}

Participants. We recruited participants from Prolific in exchange for \$2.30. Our sample was $\mathrm{N}=100$, similar to that of Studies 1a-d. Congruent with our pre-registered criteria, we removed 4 participants for providing average ratings of below 10 or above 40 . Our final sample was therefore $\mathrm{N}$ = 96 (men: 62, women:33, other: 1, Age: $M=25 \cdot 49, S D=7 \cdot 35$ ).

Procedure. Our procedure was identical to that of Study 1a with two differences. First, the sequence lengths were 2-12 and included only even sequence numbers. The was because we wanted to divide each sequence into two halves and manipulate the intensity of each half. The second difference from the tasks in Studies 1a-d was that each of the 50 trials that participants completed was divided into two conditions: high-intensity end and low-intensity end. In the high-intensity end trials, the faces in the first half of the sequence were randomly drawn only from the low intensity emotions (125 on our scale), and the second half were only randomly drawn from the high intensity emotions (26-50 on our scale). The low-intensity end trials were structured in the opposite manner, such that the first half included only high intensity faces, and the second half low intensity. We designed the task so that the low-end and high-end trials would mirror each other completely. The order of the high-end and low-end trials was random. Following the task, participants completed a survey similar to previous studies (see SM for full analysis).

\section{Results}

We first tested the three amplification hypotheses as we had done in Studies 1a-d, using identical analyses. Results suggested that participants generally estimated the emotions in the sequence as more intense than they actually were $(b=.77[.51,1.02], \mathrm{SE}=.13, t(9,814)=5.89, p<$ $.001, R 2=.06)$. Our tests of Hypotheses 2 and 3 indicated that the length of the sequence also led to increased amplification $\left(b=.25[.18, .32], \mathrm{SE}=.03, t(4,90-)=7.34, p<.001, R^{2}=.14\right)$, and that negative sequences led to a significantly stronger amplification than positive sequences $(b=.69$ [.19, 1.13], $\left.\mathrm{SE}=.24, t(4,895)=2.92, p=.005, R^{2}=.13\right)$.

Next, we evaluated the tendency for amplification in the high-intensify-end and the lowintensity-end trials. This was done by looking at the difference between participants' estimation and 
the actual sequence average as the dependent variable, and the order of high and low intensity face as the independent variable, including a by-participant and by-face-identity random variables (see Figure 5). Looking first at the intercept of the model, which is the high intensity end condition, results suggested that in trials in which the high intensity faces were presented at the end, participants estimations were significantly higher than zero $(b=2.87[1.95,3.80], \mathrm{SE}=.45, t=6.33, p<.001)$. Results also suggested that amplification was significantly higher in the high intensity end trials compared to the low intensity end trials $(b=-4.19[-4.65,-3.74], \mathrm{SE}=.22, t(4859)=-18.29, p<$ .001). To further understand the degree of amplification/de-amplification in the low intensity end condition we releveled the conditions to examine the intercept of the model which represent the difference between the low-intensity-end trials to zero. Results pointed to a significant deamplification in the low intensity end condition $(b=-1.31[-2.24,-.38], \mathrm{SE}=.45, t=-2.90, p=.01)$. There was no significant interaction between valence and the manipulation of order.

\section{Amplification as a Function of Condition and Sequence Length}

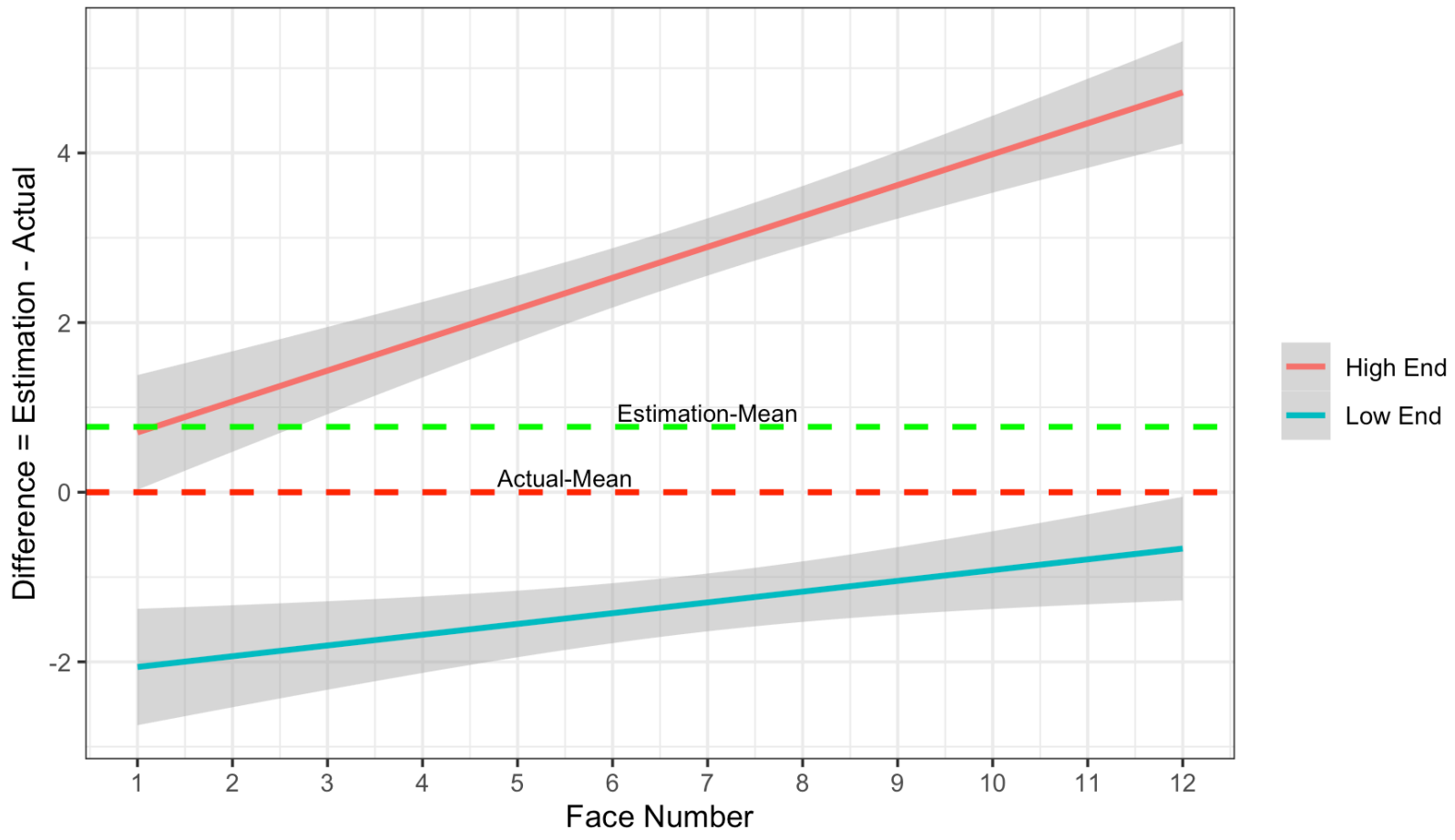

Figure 5. A summary of Study 3 results. The y-axis represents the difference between participants' estimated mean and the actual mean. Corresponding to this, the green dotted line represents the average of participants' estimated mean while the red dotted line represents the estimated mean. The $\mathrm{x}$-axis represents the number of faces that participants saw in each trial. Finally, the red or blue lines represent whether the high-intensity faces or low-intensity faces were at the end of the sequence. 
Results of Study 3 provide additional support for the importance of memory to the evaluation of sequences. We found amplification when high intensity faces were presented at the end of the sequences, and de-amplification when low intensity faces were presented at the end. Importantly, however, despite the fact that recency seemed to have a tremendous effect on evaluation, the amplification in the high-intensity end condition was larger than the de-amplification in the low intensity end conditions. This was evidenced by the fact that amplification was found across trials.

\section{Study 4: Manipulating Salience}

The goal of Study 4 was to examine the effect of memory on participants' tendency for amplification by manipulating the salience of either high or low intensity emotions. Manipulation of salience was done by adding a red square around either the high intensity or low intensity faces in a sequence. Our pre-registered hypotheses (https://osf.io/yxqem/) were that we would see amplification (H1), that amplification would increase with sequence length (H2) and that amplification would be stronger for negative emotions (H3). Finally, we hypothesized that amplification would be higher for the task in which high intensity faces were emphasized by our salience manipulation compared with when low-intensity faces were emphasized. We had no clear prediction regarding whether amplification in the control condition (no salience) would be more similar to results from the high or low intensity salience conditions $\left(\mathrm{H}_{4}\right)$.

\section{Methods}

Participants. Unlike our order manipulation in Study 3, which was likely undetected by participants, we worried that our salience manipulation would be obvious to participants if we employed a within-participants design. We therefore decided to use a between-subjects design and recruited 100 participants per each condition for a total $N=300$. Participants were recruited from prolific in exchange for $\$ 2.30$. Our initial sample was $\mathrm{N}=300$. Congruent with our pre-registered criteria, we removed 5 participants for providing average ratings of below 10 or above 40. Our final sample was therefore $\mathrm{N}=295$ (men: 119, women:175, other: 1, Age: $M=25.14, S D=8.06$ ).

Procedure. Participants were randomly assigned to one of three conditions (between-subject design): high salience, low salience, and control. In the high salience condition, participants completed a task that was similar to that of Studies 1a-d with one difference: every time a face with emotional 
intensity of more than 29 was presented, this face was presented with a red frame around it. We chose a threshold of 30 and not 25 to slightly reduce the frequency of red frames and increase the potential salience. In the low salience condition, red frames appeared around faces with an emotional intensity less than 21. The control condition was identical to Study 1a. Following the task, participants completed a survey similar to previous studies (see SM for full analysis).

\section{Results}

We first tested the three amplification hypotheses using analyses identical to those used in Studies 1a-d. Results suggested that participants generally estimated the emotions in the sequence as more intense than they actually were $\left(b=1.63[1.44,1.83], \mathrm{SE}=.9, t(29,023)=16.49, p<.001, R^{2}=\right.$ .07). The length of the sequence also led to increased amplification $(b=.36[.32, .39]$, SE $=.02, t$ $\left.(14,406)=18.09, p<.001, R^{2}=.22\right)$. Finally, negative sequences led to a significantly stronger amplification than positive sequences $\left(b=.52[.25, .79], \mathrm{SE}=.13, t(14,409)=3.69, p<.001, R^{2}=.20\right)$.

Next, we compared the magnitude of amplification between the three conditions, low intensity salience, high intensity silence, and control. We used our control condition as a baseline condition. Results suggested that the high salience condition led to significantly stronger amplification compared to the control condition $\left(b=1.39[.28,2.51], \mathrm{SE}=.56, t(291)=2.44, p=.01, R^{2}=.19\right)$. There was no significant difference between the control condition and the low salience condition $(b=.40[-.73$, 1.54], $\left.\mathrm{SE}=.58, t(291)=.69, p=.48, R^{2}=.19\right)$. One potential reason for the lack of difference between the low salience and the control condition may be that the salience of the emotional faces overrode the manipulation, and so once participants got used to the red frames, they returned to focus on the high intensity faces. To evaluate this possibility in an exploratory analysis, we tested for an interaction between the condition and the trial number. Looking first at the interaction between control and high salience, results suggested that the interaction was non-significant, such that there was no difference in the degree of amplification between the control and the high silence conditions $(b=.01[-.005$, $.038], \mathrm{SE}=.01, t(14,365)=1.44, p=.14)$. However, there was a significant interaction between trial and condition when comparing the control condition and the low salience condition $(b=.04$ [.02, .06], $\mathrm{SE}=.01, t(14,366)=3.81, p<.001)$. Further analysis of simple effects of the amplification over trial number for each of these two conditions by centering the model on different conditions suggested that there was no significant change in amplification in the control condition $(b=-.006[-.02, .01], \mathrm{SE}=$ $.01, t(14,366)=-.86, p=.38)$. However, for the low salience condition, amplification significantly 
increased with trial number $(b=.03[.02, .05], \mathrm{SE}=.01, t(14,365)=4.23, p<.001$, see Figure 6$)$. While these results do not provide direct evidence as to whether emotional salience led participants in the low salience condition to focus more on emotional faces as the task progressed, it is congruent with the notion that there was a change in the way that participants in the low salience condition were completing the task as it unfolded, while no such change was seen in the other conditions.

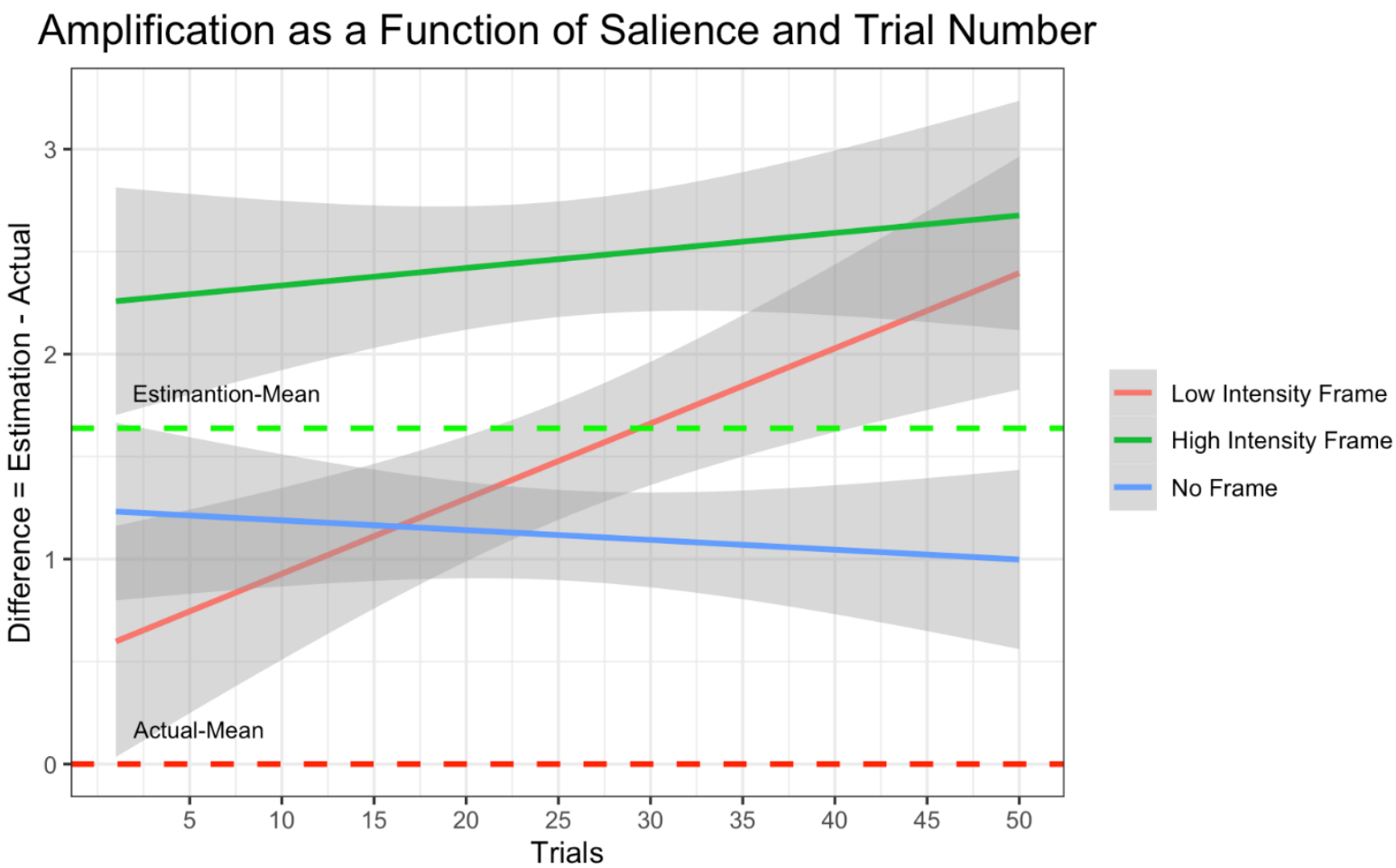

Figure 6. A summary of Study 4 results. The y-axis represents the difference between participants' estimated mean and the actual mean. Corresponding to this, the green dotted line represents the average of participants' estimated mean while the red dotted line represents the estimated mean. The $\mathrm{x}$-axis represents the trials number. Finally, the red, green and blue lines represent whether the salience manipulation of the red frames was done on the high intensity frame, low intensity trials or control condition.

Taken together, our manipulation of salience was successful in differentiating the high salience and the control condition, such that salience increased amplification. However, no difference was found in degree of amplification between the control and the low salience condition. This lack of 
differentiation could have been caused by the salience manipulation being overwhelmed by the natural salience of the high intensity faces. Exploratory analyses looking at amplification as a function of trial number in the low salience condition indicated that amplification increased with trial number, pointing to the possibility that as participants in the low salience condition habituated to the salience of red frames, the effect of high intensity emotions became more salient.

\section{Study 5: Examining Amplification Over and Above Nonlinearity in Emotion Perception}

Findings from this study series support the idea that when perceivers estimate the mean emotion of a sequentially presented series of emotion expressions, they systematically over-estimate the mean. Thus far, our analysis of underlying mechanisms has focused on differential memory for more emotional (intense, salient) stimuli. However, another explanation of the apparent bias we observe is that it arises from a nonlinearity in the perception of the emotion faces that we presented. More precisely, it is possible that there is a perceptual asymmetry in how our lower-valence faces are perceived relative to our higher valence faces, such that a middle-valence faces are seen as more similar to higher-valence faces than to the lower-valence faces. Participants would then be more likely to confuse the middle-valence faces with the higher-valence ones than the lower valence ones, leading to an amplification in their estimation of the average. This explanation would entail that it is the perceptual characteristics of the stimulus space, rather than changes in memory that drive the observed amplification effects. Given this concern, we used a computational modeling approach to separately quantify the psychophysical similarity between faces, and used this similarity data to estimate what biases in memory for ensembles would be expected based on similarity alone.

To achieve this goal, we first empirically tested how people perceived distances between emotional intensities at different points of our emotional scale. We then built on an existing computational model (Robinson \& Brady, 2021) that was designed to simulate ensemble memory with specific attention to non-linearity in similarity, by comparing three hypothetical models of ensemble coding: A baseline model, that only incorporated nonlinearity in similarity, a recency model that was based on the baseline model but also assumed stronger weight in memory for more recent items, and an amplification model that was based on the recency model but added an assumption of increased weight to more emotional faces. We used the results of Study 3 to compare these three models' fit.

\section{Method}


The current project involved two steps. In the first step, we ran a similarity task to examine non-linearity in participants' estimation of similarity at different locations of the scale. In the second step we implemented the findings from our similarity study and compared the fit of three hypothetical models using the data from Study 3.

Participants. We recruited participants from Prolific in exchange for \$2.70. We aimed for a similar number of participants as in our other studies. No participants were excluded from the study. Our final sample was $\mathrm{N}=100$ (men: 37, women:62, other: 1, Age: $M=35.99, S D=12.69$ ).

Procedure. Recent work by Schurgin, Wixted and Brady (2020) took a computational modeling approach to delineate the effects of the psychophysical similarity of stimuli on well-known memory phenomena in visual memory. These authors found that once the psychophysical similarity of stimuli was taken into account, many purported memory phenomena were in fact reducible to the perceptual confusability of stimuli. To evaluate similarity in emotional perception we modified the similarity task that was used by Schurgin and colleagues. In each trial, participants saw two faces on the screen and were asked to evaluate to what degree these two faces were similar to each other on a 1-7 scale, 1 - not similar at all, 7- very similar. Participants had as much time as they needed to make their selection The similarity between two faces was measured using a seven-point Likert scale, where Smin $=1$ and Smax $=7$. To generate the psychophysical similarity function, we simply normalized these data to range from o to 1 , giving a psychophysical similarity metric, such that $\mathrm{f}(\mathrm{x})=((\mathrm{Sx}-$ Smin)/(Smax - Smin )). In order to cover the whole 1-50 scale, one every five faces was selected and compared to all other faces in increments of 5 . For example, a face of emotional intensity 1 was compared to: $1,5,10,15,20,25,30,35,40,45,50$. Completing all comparisons within a certain scale required conducting 66 comparisons. In each study participants completed 264 (66 X 4) comparisons, which meant that each participant completed all possible comparisons in four out of the eight faceemotion continua: 4 identities X 2 valences (neutral-to-happy and neutral-to-angry). The 4 identities that were chosen randomly for each participant.

\section{Results}

Similarity Analysis. Our first analysis involved evaluating the linearity in the relationship between the actual distance between the faces and the estimated distance. We conducted a mixed model analysis looking at actual item similarity predicting perceived similarity. To evaluate non linearity we introduced a second and third-order polynomial in addition to the linear slope of the 
model using the poly function in $\mathrm{r}$ (R Core Team, 2013). Results suggested that both the linear term $(b$ $=-.24[.28,2.51], \mathrm{SE}=.002, t(26,297)=-85.72, p<.001)$, the second-order polynomial $(b=-.006$ $[.28,2.51], \mathrm{SE}=.002, t(26,297)=-2.77, p=.01)$, and the third-order polynomial $(b=02[.28,2.51]$, $\mathrm{SE}=.001, t(26,297)=15.02, p<.001)$ were significantly associated with the data. Model comparisons of the current model (AIC =-1279.92) with both a model which had only a linear and a second-degree polynomial slope (AIC = -1068.48) and only a linear model slope (AIC =-897.63), suggested that the model with the third-degree polynomial slope was the strongest (Figure 7).

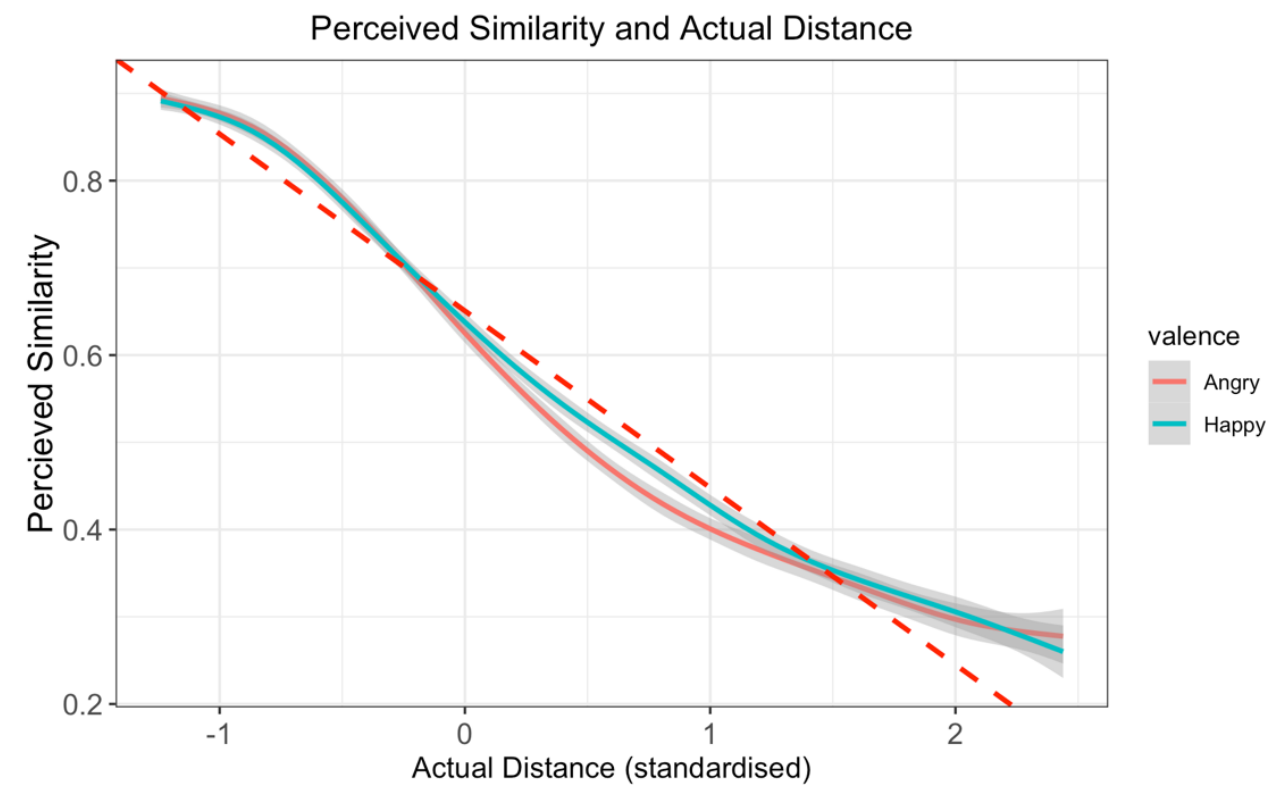

Figure 7. Results from the similarity analysis in Study 5 . The $\mathrm{x}$-axis represents the difference between item 1 and item 2 . As can be seen by the changes in the degree of slope, both shorter distances and long distances were perceived as more similar compared to distances between these two extremes.

Comparing Ensemble Coding Models. Having established non-linearity in similarity perceptions, we then took a computational modeling approach to validate that indeed that the amplification found in our studies did not stem from nonlinearity and perception of similarity. We adapted a recently developed model for ensemble memory (Robinson \& Brady, 2021), which is the first computational model to make high-precision predictions of performance in continuous report memory ensemble tasks. In this work, we treat this memory for ensembles as a measurement model; that is, as explained, we use it to formally separate effects of psychophysical similarity from amplification memory biases. 
This model of memory for ensembles postulates that each stimulus evokes a distributed pattern of activation over feature values, and ensembles are computed by pooling over these patterns of activation at a relatively early perceptual stage of processing. Critically, within this modeling framework, the pattern of activation evoked by each stimulus depends on the psychophysical similarity of features to items held in memory, such that feature values that are more like items held in memory receive a higher boost in activation. This model directly links psychophysical similarity to memory processes by postulating that the patterns of activation elicited by each stimulus determines how familiar that feature, and similar features will feel. For instance, if a task requires remembering a certain emotional intensity, the specific intensity will evoke a very strong familiarity signal, but so will similar emotional expressions. Finally, in line with mainstream signal detection models of memory (Wickens, 2001), the model posits that ensemble memory representations are corrupted by noise and that the signal-to-noise ratio depend on factors that determine the top-down upweighting of features of individual items (e.g., manipulation of memory load, delay, or presentation format). Formally, the most straightforward version of this model for ensembles is given by the following equation:

$$
R_{E N S}=\operatorname{argmax}\left(\left(\sum_{i=1}^{N} f(x)_{i} d^{\prime}\right)+\sigma_{\text {Noise }}\right)
$$

where $R_{E N S}$ is the reported feature on the ensemble task (i.e., which face is chosen), $N$ is the total number of items in the ensemble memory array, $f(x)$ is the psychophysical similarity function of item $i$ (i.e., it captures how similar each of the 50 faces are to item $i$; we describe the measurement of this below). $d^{\prime}$ is a free parameter that determines the level of activation of each feature value for each item. Note that this version of the model postulates that on average, each item in the sequence generates the same familiarity signal, meaning that $d^{\prime}$ is the same value for each item in the sequence (that is, the model only has one free parameter $d^{\prime}$ ). $\sigma_{N o i s e}$ is a fixed amount of noise ${ }^{1,}$ and $\operatorname{argmax}$ denotes the decision rule that memory reports are based on the feature that generates the maximum familiarity signal. More precisely, the argmax argument is taken over a vector of random variables $\left(X_{1}, X_{2}, X_{3}, \ldots X_{50}\right)$, where each random variable is one of the fifty possible faces on the self-report scale, each of which is distributed according to the model equation given in the parentheses. We refer to the above model as the Baseline model because it assumes that 1) the familiarity of the ensemble is solely determined by its psychophysical similarity, and that on, on average, 2) there is equal weighting of

\footnotetext{
${ }^{1}$ Noise is set to one standard deviation of a Gaussian distribution, consistent with a signal detection model.
} 
each item in memory - that is, there are no sequential or amplification effects on memory (i.e., no recency or exaggeration of the impact of negative faces).

The second variant of the ensemble model we use is the Recency model (Robinson \& Brady, 2021), which postulates that memory performance in the sequential paradigm is determined by psychophysical similarity as well as higher weighting of more recent items in memory (recency effects). In line with extant recency models of memory, the recency weights are quantified with a normalized exponential function (without base $e$ ) defined over the serial position of each stimulus in the sequence (Tong et al., 2019). The recency model is given by the following equation:

$$
\begin{gathered}
R_{E N S}=\operatorname{argmax}\left(\left(\sum_{i=1}^{N} f(x)_{i} d^{\prime} w_{i}^{\text {Recency }}\right)+\sigma_{\text {Noise }}\right) \\
w_{i}^{\text {Recency }}=\frac{r^{i}}{\sum_{i=1}^{N} r^{i}}
\end{gathered}
$$

where $w_{i}^{\text {Recency }}$ is the recency weight of the ith item in the sequence, and $r$ is a free parameter that the rate of prioritization as a function of the serial position of a stimulus (Tong et al., 2019). This version of the model, therefore, has two free parameters, $d^{\prime}$ and $r$. The critical point to note is that Equations 1 and 2 are identical except that Equation 2 can also capture higher weighting of more recent items. Thus, a comparison of these models provides insight into whether there is evidence for higher prioritization of more recent items in the sequence once psychophysical similarity is taken into account. Given prior evidence for recency effects in ensemble tasks, as well as the studies reported above, we expect the recency model to outperform the baseline model.

The final model we refer to as the Amplification Recency model. This model of ensembles postulates that in addition to effects of psychophysical similarity and recency on memory, there is also amplification of emotional faces. As noted, we use this model as a measurement model $^{2}$, to formally separate possible effects of amplification from psychophysical similarity and recency. Accordingly, in line with our behavioral results, we make the simplifying assumption that recency and amplification combine independently to bias memory, and that amplification increases exponentially as a function of a face's emotional extremeness. The model equation is shown below.

\footnotetext{
${ }^{2}$ This entails that we do not assume that is the best descriptive model of amplification, but rather use it to quantitatively separate amplification biases from psychophysical similarity and recency effects.
} 


$$
\begin{gathered}
R_{E N S}=\operatorname{argmax}\left(\left(\sum_{i=1}^{N} f(x)_{i} d^{\prime} w_{i}^{\text {Recency }} w_{i}^{\text {Amplification }}\right)+\sigma_{\text {Noise }}\right) \\
w_{i}^{\text {Amplification }}=e^{A(j / 50)}
\end{gathered}
$$

As shown in the above equations, the $w_{i}^{\text {Amplification }}$ weight is an exponential function of the item's emotionality, which is denoted by $j$ (1-50), and a free parameter $A$ Note that larger values of $A$ indicate higher weighting of more emotional faces, and we constrained $A$ to be non-negative (zero inclusive) to capture the fact that there may be no amplification (when $A$ equals zero). Thus, this model has three free parameters, $d^{\prime}, r^{\prime}$ and $A$. As before, the Amplification Recency model is like the Baseline and Recency model except that it posits that memory biases are jointly determined by psychophysical similarity, recency effects and amplification of more extreme faces. Therefore, a comparison of the Amplification Recency model with the Baseline and Recency models provides direct insight into whether there are amplification memory biases once psychophysical similarity and recency effects are taken into account.

Prior to model fitting, we evaluated each of these models using parameter and model recovery analysis (for discussion of best practices in cognitive modeling see: Heathcote et al., 2015). In our model recovery analysis, we found that Akaike Information Criterion (AIC) is the metric that recovered the true data generating model, therefore, we focus on this metric for the model comparison. We also found that we were able to recover the true data generating model, as long as the presentation of emotional faces was tightly controlled in the sequence. Study 3 was a good fit for this criteria because the order of the high and low intensity was manipulated, which, based on the model recovery analysis, allows us to differentiate between the competing models. . In studies in which recency is not manipulated, it is hard to differentiate recency from amplification (likely because these effects wash each other out). We fitted these three models to the data using a log-likelihood minimization function and compared their fit using AIC (see Table 2). Results suggested that overall, the amplification model yielded the best fit to the data, providing additional support for amplification over and above recency and non-linearity. More specifically, for trials with happy faces, the recency model was actually the one performing the best out of all models, the second best performing model was the recency amplification model and the last was the baseline model. For trials with angry faces, the recency amplification model was the strongest fit, with recency second and baseline third. It is important to note that all of the models were fitted to the data of Study 3, in which for positive faces, no significant amplification was found. These results do not reflect the general trend in many of our 
other studies and may have been driven by the recency manipulation. Regardless, the fact that the amplification model was the best predictor of the data overall is encouraging. These results provide converging support for the view that amplification is not driven by the psychophysical similarity of our stimulus set, but is indeed driven by memory bias.

Table 2. AIC comparisons for three models, the baseline model, the recency model and the amplification model for happy face, angry faces and both.

\begin{tabular}{|l|l|l|l|}
\hline & \multicolumn{3}{|c|}{ AIC } \\
\hline \multicolumn{1}{|c|}{ Model } & \multicolumn{1}{|c|}{ Happy } & \multicolumn{1}{|c|}{ Angry } & Total \\
\hline Baseline Model & 16275 & 16561 & 32835 \\
\hline Recency Model & 16119 & 16422 & 32540 \\
\hline Recency Amplification Model & 16135 & 16386 & 32522 \\
\hline
\end{tabular}

\section{Study 6: Amplification in the Evaluation of Emotional Videos}

The goal of Study 6 was to generalize the findings from Studies 1-4 and examine them in more naturalistic interactions. To achieve this goal, we used data from the Stanford Emotional Narratives Dataset (Ong et al., 2019), in which observers watched and provided emotional ratings of videos of a diverse set of targets telling personal emotional stories. Participants were asked to provide two types of ratings in response to each video, the first was a continuous evaluation of the degree of negativity and positivity of each video, and the second was a global evaluation of the target emotionality after watching the whole video. These two measurements allowed us to compare between the average of participants' real-time emotional evaluations and their post-video global evaluations. Based on findings in the previous studies, we hypothesized that participants' global evaluations would be stronger compared to the averages of their continuous evaluations (H1) and that amplification would be stronger for videos with negative compared to positive narratives (H2). Given that videos did not 
differ significantly in length, this study was not suitable to validate the association between length and amplification, but it was tested nevertheless showing no change (See SM for full analysis).

\section{Methods}

Target Videos. Target videos were collected as part of the Stanford Emotional Narratives Dataset (SEND, Ong et al., 2019). Targets were brought into the lab and were asked to think of the three most positive and three most negative events that they would feel comfortable sharing in front of a video camera. Recording was self-paced: The experimenter left the target alone in the room, and targets were allowed to talk for as long as they wanted about each event. After targets finished recording the videos, they were asked to fill out several personality and demographic surveys. During this time, the experimenter processed the videos by transferring them from the camera onto the computer and prepared the next part of the experiment. After targets finished the surveys, they were then shown each video that they recorded. While watching each video, they were asked to provide continuous ratings of how they felt as they were telling their story. These ratings were collected using a visual analog scale divided into a hundred points, ranging from "Very Negative" (-1) to "Very Positive" $(+1)$. The ratings on the scale were sampled every 0.5s. Participants gave their consent to use the videos in future experiments. The subset of video clips selected for the SEND were all consented for research use.

Of the videos that were produced by participants, 193 were selected containing 49 unique targets (Gender: men=20, women=27, other: 2; Age: $M=24.8, S D=9.6$, Race: East Asian $=6$, South Asian $=3$, Black $=2$, Hispanic $=4$, Middle Eastern $=1$, White $=16$, Mixed $=13$, Other $=4)$. This set was chosen such that: (i) the target's face was always in the camera, (ii) the clips did not contain sensitive content (e.g. mental health, suicide), and (iii) the clips were emotional, and had some narrative flow (rather than stream of consciousness or rambling). The clips were also cropped for length, such that the final clips lasted on average 2 minutes 15 seconds. Videos were divided into 4 valence categories by the original authors, which we retained in this study (Figure 8). After transforming video ratings to be on a 0-100 scale (o-very negative, 50-neutral, 50- very positive) videos were divided to four categories. Positive videos included videos that were rated by targets on average as higher than 6o, with a minimum rating of .40 $(n=62)$. Negative videos' average were lower than 40 with a max rating of $60(n=33)$. Neutral videos were videos that had a max rating of 60 and 
min rating of $40(n=30)$. All other videos were categorized as mixed (68). See original paper for full description of the videos (Ong et al., 2019).

Participants. We use the terms observers to describe participants who were collected separately at a later date and were asked to provide their evaluation of the target's emotionality. Observers were recruited as part of the SEND database on Amazon Mechanical Turk to watch either videos clips and provide ratings of how the target in the video felt (for details, see Ong et al., 2019). Observers saw each video along with a continuous sliding scale underneath that was designed for continuous emotional ratings. They were asked to dynamically adjust the scale as the video played to capture the emotional intensity of the target at each time point. The analog scale was divided into 100 points (o-very negative, 50-neutral, 100-very positive) and sampled every 0.5s. Severn hundred participants were recruited with the goal of getting at least 20 participants rating each video. Each participant watched 8 videos. The final recruited sample was 695 participants, with 11 additional participants being removed for failing to correctly answer two comprehension checks. Of the remaining 684 participants, we divided the continuous data to windows of 2 seconds and removed any observer ratings for videos that included less than 5 ratings. This elimination standard was different from that of the original researchers who only removed participants who provided zero continuous ratings. We believe that such criteria are a more conservative comparison for the analysis. However, using the original authors' criteria does not change the significance of the results. Our final sample therefore was $\mathrm{N}=565$ (age: $M=37.23, S D=11.23$, gender: female $=279$, male $=254$, undefined = $32)$.

Data Reduction. One concern that may be raised when comparing the continuous and postrating measures is that the continuous rating included the beginning of the videos in which participants did not change their ratings, which meant that their rating was de-facto neutral. Keeping these ratings may artificially reduce the overall average of the continuous rating and further emphasize the amplification. To avoid this issue, we cut the continuous ratings to start only when observers made their first change to the rating, thus removing sections in which the rating was neutral. We then averaged each continuous rating from the point in which participants made their first rating to the end of the video.

Measures. Observers provided two types of ratings in response to each video. The first rating was a continuous rating on a $0-100$ scale, $\mathrm{o}$ indicating very negative, 50 indicating neutral, and 100 
indicating very positive. Ratings were sampled every 0.5 second. After watching the video, participants were asked to rate the degree of the target positivity and negativity using two ratings on a 1-7 scale (1-neutral, 7-very emotional), one for positive emotion and one for negative emotions. Because the correlation between positive ratings and negative ratings was very strong $(r=-.79[-.75,-$ .82], and in order to compare the continuous ratings to the post-ratings, we averaged between the positive and negative ratings post-ratings, creating one scale for post-ratings, 1-very negative to 7very positive. To compare the post-ratings with the continuous ratings we converted the continuous rating to a 1-7 scale by dividing it by 100, multiplying by 6 and adding 1 . With this transformation, 100 on a continuous scale was equal to 7 and o was equal to 1 .

\section{Results}

One of the challenges of the current analysis is that amplification may be driven by the fact that continuous and post-measures were evaluated with different scales. In order to account for differences that may have been caused by different scales, we treated the difference between postrating and continuous rating of the neutral videos as our baseline comparison. Although using neutral videos as a comparison may not solve issues that stem from differences in nonlinearity of the two scales, it can account for baseline differences.

In order to evaluate amplification, we created a difference score between participants' postrating and their continuous rating such that a positive value indicates that the post-rating was more positive and a negative value indicates that the post-rating was more negative. We then conducted a mixed model analysis using the difference score as our dependent variable and the valence of the video as the dependent variable. Similar to our previous models, we also included by-participant and by-video random intercepts. As previously mentioned, we set our model to use the neutral videos as the baseline comparison (the intercept of the model).

Results suggested that for the neutral videos, the intercept of the model, the difference between participants' post-ratings and their continuous rating was not different from zero $(b=.16$ [$.06, .39], \mathrm{SE}=.11, t(191)=1.39, p<.001)$ providing another indication that neutral condition is a reasonable baseline for comparison. Looking at the negative videos, the difference between the postrating and the continuous ratings was significantly more negative compared to the difference in the neutral condition $(b=-.92[-1.24,-.60], \mathrm{SE}=.16, t(189)=-5.66, p<.001)$. On the other hand, and also congruent with the tendency for amplification, the difference between post-ratings and 
continuous ratings in the positive videos was significantly more positive than the neutral videos ( $b$ $=.70[.42 .98], \mathrm{SE}=.16, t(189)=4.87, p<.001$, Figure 8$).$

To further compare the difference between post-ratings and continuous ratings in the positive and negative conditions we multiplied the difference score between the post-ratings and the continuous ratings by -1 , thus allowing us to compare the difference score in the positive and negative ratings. We then conducted a mixed model analysis similar to the one above, this time centering the model on the negative videos. Results suggested that amplification in the negative videos was not significantly different in absolute magnitude than that in the positive videos $(b=.10[-.16, .37], \mathrm{SE}=$ $.14, t(189)=.73, p=.46)$.

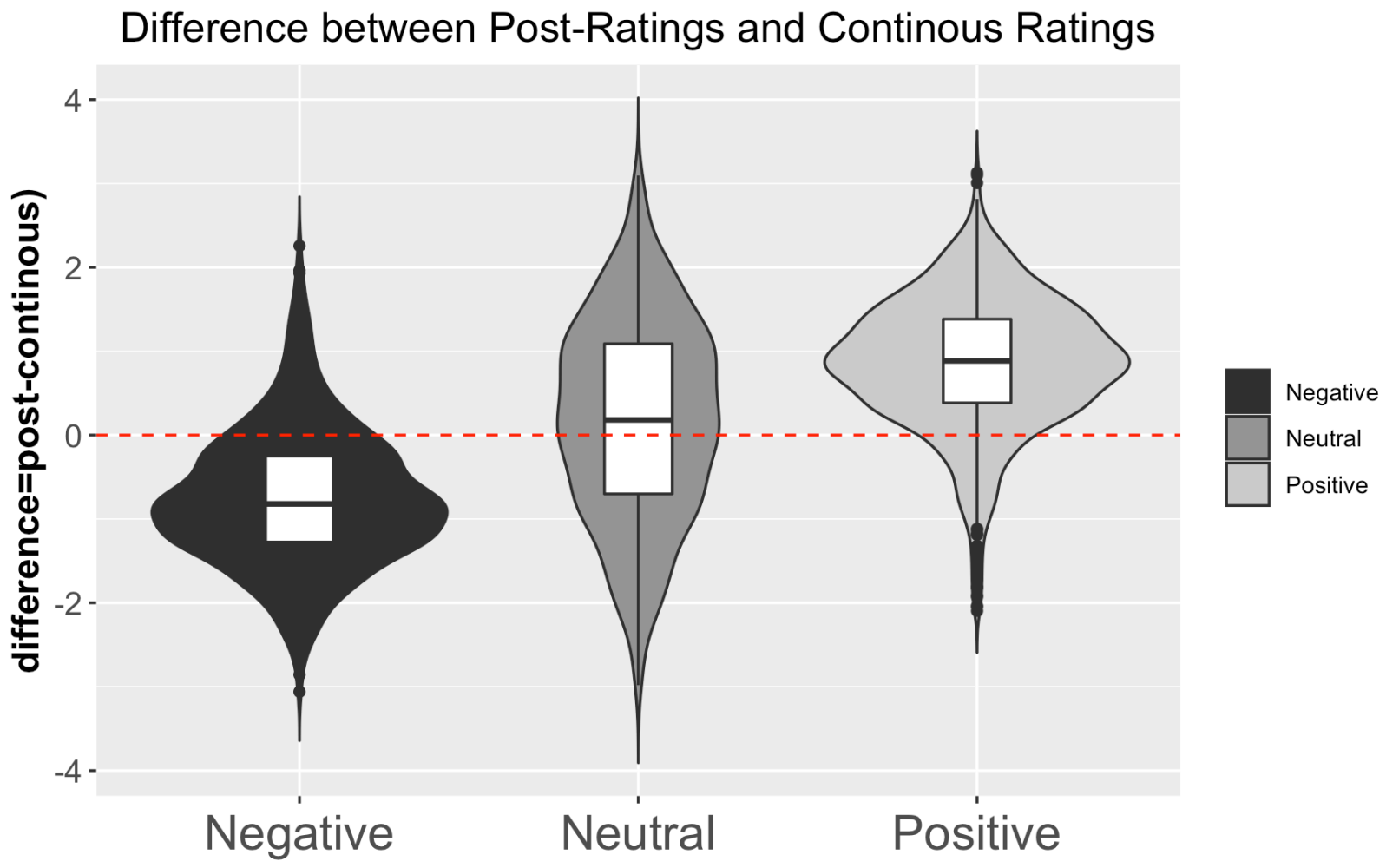

Figure 8. The difference between post and continuous ratings for the three types of videos, neutral, negative, and positive. A positive number indicates that the post rating was more positive than the average continuous rating and a negative number indicates that the post ratings was more negative than the continuous rating.

To summarize, analysis of the SEND videos pointed to an amplification effect that was similar to the one evident using sequences of static emotion expressions in Studies 1-4. Results with the 
videos pointed to no amplification in the neutral videos, but we did find amplification in the positive, the negative, and the mixed videos. There was no difference between amplification in the positive and the negative videos.

\section{General Discussion}

Social interactions frequently involve a certain degree of emotionality, which is reveled to the perceiver over time. But how do people rapidly estimate emotional sequences unfolding over time, and are they are accurate in doing so? In a series of 9 studies, using a diversity of stimuli and analysis approaches, we showed that people tended to overestimate the average emotionality of emotional sequences. We also showed that longer sequences were further amplified and that this was stronger for sequences of negative emotions. Our memory analyses suggested that people tended to better remember both recent and stronger emotional expressions in the sequence, and that manipulating memory by changing recency or salience of certain faces, led to corresponding changes in amplification. Using a computational approach, we also showed that a model which assumed increased memory for stronger emotional expressions performed better in predicting the data than other models without such amplification. Finally, we provided initial evidence for amplification in the evaluation of naturally evolving emotional stories using the perception of naturalistic videos.

Our studies shed light on an important aspect in social interactions that impacts our daily decisions and behaviors. In almost any of our social interactions our evaluations of our partner's emotions impact the way we respond and the actions we take in return. Accurate evaluations of our partner's emotions are even more important when certain crucial decisions are based in perceivers evaluation of emotionality. Take for example a job interview, in which candidates are evaluated in part on their passion to the job, or medical decisions that are based on doctors' perception of the degree to which their patients are in pain. All of these should be impacted by amplification.

\section{Mechanisms Underlying the Sequential Amplification Effect}

One suggested mechanism for the sequential amplification effect is preferential memory for more emotional faces. Increased salience of emotional faces leads to greater likelihood that they could be retrieved when evaluating the sequence, which means that stronger emotions are more likely to be weighted in the evaluation of the sequence. In the following set of studies, we indeed showed that participants tended to remember emotional faces better, and that enhancing memory by changing the order of the high intensity faces, or by increasing their salience led to increased amplification. 
Despite this evidence, one potential alternative description of the way sequences are integrated may point to a different mechanism for amplification. It is possible that observers maintain a running average of a sequence as it unfolds. If this is the case, amplification cannot be driven by memory but rather by some bias in the way that some faces are added to the running average. Previous research on sequential ensemble coding has explored this possibility in a set of experimental studies (for further discussion, see Tong et al., 2019). One finding that goes against the running average theory is the fact that recent items in the set more strongly impact the evaluation of the sequence average compared to less-recent items, whereas according to a running average account there is no reason to assume that recent items should be weighted differently. This of course does not exclude the option that participants are trying to maintain a running average, but it does detract from the likelihood that a running average account alone can explain our findings. Future work should attempt to examine bias in running averages by forcing participants to report a running average and examine whether amplification is found in these situations (see Tong et al., 2019).

Another reason to think that bias in running average is likely not the primary mechanism that drives amplification are recent findings that point to amplification in ensemble coding of items occurring concurrently. In the past few years, amplification has been found in the perceptions of circle sizes (Kanaya et al., 2018), orientation (Lakovlev \& Utochkin, 2020), and even emotions (Goldenberg et al., 2020), the most recent seems to be mediated by increased attention to more emotional faces. One question is therefore how to integrate these findings under a single spatio-temporal model. It is possible that when people are looking at a crowd or an array of circles, they sequentially scan people in the crowd, spending more time attending emotional faces and therefore leading to their over representation in memory. Further research, however, is needed to validate such model to show that the tendencies for sequential and concurrent amplification are driven by similar mechanisms.

\section{Limitations and Future Directions}

Our experiments revealed a tendency for amplification that seems to be relevant to many aspects of social behavior. Nevertheless, these studies leave open several questions regarding how amplification manifests itself in natural social interactions outside the lab.

One limitation relates to the idea that asking participants to evaluate the emotional average of a sequence is dissimilar to the way such evaluations are done naturally. Research suggests that people both naturally segment continuous stimuli (Zacks \& Swallow, 2007), and extract average features 
from a set even when are not asked to do so, these studies were mostly done on ensemble perception of items occurring concurrently (Dubé et al., 2014; Oriet \& Hozempa, 2016). It is important to note that in Study 6 participants were not asked to evaluate the sequence on average but rather to provide a global evaluation of emotionality, leading to similar results. However, despite these findings, it is definitely possible that sequences are naturally aggregated in a way that differ from the mean. Future work should be done to further examine what people do when asked to naturally evaluate emotionality of expression sequences.

A second limitation relates to the fact that many of the studies reported here involve evaluation of emotional sequences of multiple pictures that were uniformly drawn from an array of expressions. In real life, when people estimate emotion expressions unfolding over time, emotions tend to cluster together. In other cases, a person's emotionality can only be deciphered from a single emotional expression in an otherwise non-emotional exchange. One question that is raised is therefore how does the distribution of emotions in a sequence impact the evaluation of a person's emotionality? A second question is whether when emotions are clustered in a way that is more similar to their natural occurrence, whether amplification still exists. Future work should examine these questions.

A third limitation relates to the fact that in many of our studies (except Study 6), participants evaluated sequences expressed in clear facial expressions by white men, while participants' themselves were not experiencing any emotion. People's ability to aggerate multiple emotions is likely to depend on target attributes such as gender, race, age and culture, but also on perceivers' emotional state. Further studies should manipulate these aspects and examine their contribution to amplification. It may also be the case that amplification occurs as a result of exposure to various types of emotional expressions, not only faces. For example, much of our exposure to emotions on social media is done sequentially via text. Further understanding how people integrate this sequential information of various sources and kinds can shed light on the way people react and respond to social information.

To conclude, this project reveals an extremely important aspect of social cognition that is central to many social interactions. But, there is no reason to assume that amplification in the evaluation of sequences applies only to emotions. Its possible that any domain in which perceivers are exposed to sequences, and some items in the sequence are more salient than others, we should 
assume the occurrence of amplification. Take for example a sequential exposure to someone's moral actions. Assuming that we are more likely to remember immoral actions, we should therefore evaluate a person as more immoral than they actual are. Further understanding of how people integrate sequential information of various kinds is therefore likely to reveal other biases in the way we understand our social world.

\section{References}

Albrecht, A. R., \& Scholl, B. J. (2010). Perceptually averaging in a continuous visual world. In Psychological Science (Vol. 21, Issue 4, pp. 56o-567). https://doi.org/10.1177/o956797610363543

Alvarez, G. A. (2011). Representing multiple objects as an ensemble enhances visual cognition. Trends in Cognitive Sciences, 15(3), 122-131.

https://www.sciencedirect.com/science/article/pii/S1364661311000040

Cojuharenco, I., \& Ryvkin, D. (2008). Peak-End rule versus average utility: How utility aggregation affects evaluations of experiences. Journal of Mathematical Psychology, 52(5), 326-335. https://doi.org/10.1016/j.jmp.2008.05.004

D’Argembeau, A., \& Van der Linden, M. (2007). Facial Expressions of Emotion Influence Memory for Facial Identity in an Automatic Way. Emotion, 7(3), 507-515. https://doi.org/10.1037/1528$3542 \cdot 7 \cdot 3 \cdot 507$

Dubé, C., Zhou, F., Kahana, M. J., \& Sekuler, R. (2014). Similarity-based distortion of visual short-term memory is due to perceptual averaging. Vision Research, 96, 8-16. https://doi.org/10.1016/j.visres.2013.12.016

Eimer, M., \& Holmes, A. (2007). Event-related brain potential correlates of emotional face processing. Neuropsychologia, 45(1), 15-31. https://doi.org/10.1016/j.neuropsychologia.2006.04.022

Elias, E., Dyer, M., \& Sweeny, T. D. (2017). Ensemble perception of dynamic emotional groups. Psychological Science, 28(2), 193-203. https://doi.org/10.1177/0956797616678188

Fiedler, K. (2000). Beware of samples! A cognitive-ecological sampling approach to judgment biases. Psychological Review, 107(4), 659-676. https://doi.org/10.1037/0033-295X.107.4.659

Fredrickson, B. L. (2000). Extracting meaning from past affective experiences: The importance of 
peaks, ends, and specific emotions. Cognition and Emotion, 14(4), 577-606. https://doi.org/10.1080/026999300402808

Gallegos, D. R., \& Tranel, D. (2005). Positive facial affect facilitates the identification of famous faces. Brain and Language, 93(3), 338-348. https://doi.org/10.1016/j.bandl.2004.11.001

Goldenberg, A., Sweeny, T. D., Shpigel, E., \& Gross, J. J. (2019). Is this my group or not? The role of ensemble coding of emotional expressions in group categorization. Journal of Experimental Psychology: General. https://doi.org/10.1037/xgeoooo651

Goldenberg, A., Weisz, E., Sweeny, T., Cikara, M., \& Gross, J. (2020). The crowd emotion amplification effect. Psychological Science. https://doi.org/10.31219/osf.io/cn6qy

Gosling, S. D., Rentfrow, P. J., \& Swann, W. B. (2003). A very brief measure of the Big-Five personality domains. Journal of Research in Personality, 37(6), 504-528. https://doi.org/10.1016/Soog26566(03)00046-1

Haberman, J., Harp, T., \& Whitney, D. (2009). Averaging facial expressions over time. Journal of Vision, 9(11), 1-22. https://doi.org/10.1167/9.11.1

Haberman, J., \& Whitney, D. (2007). Rapid extraction of mean emotion and gender from sets of faces. Current Biology, 17(17), R751-R753. https://doi.org/10.1016/J.CUB.2007.06.039

Heathcote, A., Brown, S. D., \& Wagenmakers, E. J. (2015). An introduction to good practices in cognitive modeling. In An introduction to model-based cognitive neuroscience (pp. 25-48). Springer Scince.

Huang, J., \& Sekuler, R. (2010). Distortions in recall from visual memory: Two classes of attractors at work. Journal of Vision, 10(2), 1-27. https://doi.org/10.1167/10.2.24

Hubert-Wallander, B., \& Boynton, G. M. (2015). Not all summary statistics are made equal: Evidence from extracting summaries across time. Journal of Vision, 15(4), 5-5. https://doi.org/10.1167/15.4.5

Hughes, M. E., Waite, L. J., Hawkley, L. C., \& Cacioppo, J. T. (2004). A short scale for measuring loneliness in large surveys: Results from two population-based studies. Research on Aging, 26(6), 655-672. https://doi.org/10.1177/0164027504268574 
Jackson, M. C., Linden, D. E. J., \& Raymond, J. E. (2014). Angry expressions strengthen the encoding and maintenance of face identity representations in visual working memory. In Cognition and Emotion (Vol. 28, Issue 2, pp. 278-297). https://doi.org/10.1080/02699931.2013.816655

Jackson, M. C., Wolf, C., Johnston, S. J., Raymond, J. E., \& Linden, D. E. J. (2008). Neural correlates of enhanced visual short-term memory for angry faces: An fMRI study. PLoS ONE, 3(10). https://doi.org/10.1371/journal.pone.0003536

Jackson, M. C., Wu, C. Y., Linden, D. E. J., \& Raymond, J. E. (2009). Enhanced visual short-term memory for angry faces. Journal of Experimental Psychology: Human Perception and Performance, 35(2), 363-374. https://doi.org/10.1037/aoo13895

Juni, M. Z., Gureckis, T. M., \& Maloney, L. T. (2012). Effective integration of serially presented stochastic cues. Journal of Vision, 12(8), 1-16. https://doi.org/10.1167/12.8.12

Kahneman, D., Fredrickson, B. L., Schreiber, C. A., \& Redelmeier, D. A. (1993). When more pain is preferred to less: Adding a better end. Psychological Science, 4(6), 401-405. https://doi.org/10.1111/j.1467-9280.1993.tboo589.x

Kanaya, S., Hayashi, M. J., \& Whitney, D. (2018). Exaggerated groups: Amplification in ensemble coding of temporal and spatial features. Proceedings of the Royal Society B: Biological Sciences, 285(1879). https://doi.org/10.1098/rspb.2017.2770

Kaufmann, J. M., \& Schweinberger, S. R. (2004). Expression influences the recognition of familiar faces. Perception, 33(4), 399-408. https://doi.org/10.1068/p5083

Lakovlev, A. U., \& Utochkin, I. S. (2020). Roles of saliency and set size in ensemble averaging. Attention, Perception, and Psychophysics, 83, 1251-1262. https://doi.org/https://doi.org/10.3758/s13414-020-02089-w

Langner, O., Dotsch, R., Bijlstra, G., Wigboldus, D. H. J., Hawk, S. T., \& van Knippenberg, A. (2010). Presentation and validation of the radboud faces database. Cognition and Emotion, 24(8), 13771388. https://doi.org/10.1080/02699930903485076

Lee, H. J., \& Cho, Y. S. (2019). Memory facilitation for emotional faces: Visual working memory tradeoffs resulting from attentional preference for emotional facial expressions. Memory and Cognition, 47(6), 1231-1243. https://doi.org/10.3758/s13421-019-00930-8 
Luck, S. J., \& Vogel, E. K. (1997). The capacity of visual working memory for features and conjunctions. Nature, 39o(1996), 279-281.

Mattick, R. P., \& Clarke, J. C. (1998). Development and validation of measures of social phobia scrutiny fear and social interaction anxiety. Behaviour Research and Therapy, 36(4), 455-470. https://doi.org/10.1016/Sooo5-7967(97)10031-6

Ong, D. C., Wu, Z., Zhi-Xuan, T., Reddan, M., Kahhale, I., Mattek, A., \& Zaki, J. (2019). Modeling emotion in complex stories: The Stanford Emotional Narratives Dataset. IEEE Transactions on Affective Computing. https://doi.org/10.1109/taffc.2019.2955949

Oriet, C., \& Brand, J. (2013). Size averaging of irrelevant stimuli cannot be prevented. Vision Research, 79, 8-16. https://doi.org/10.1016/j.visres.2012.12.004

Oriet, C., \& Hozempa, K. (2016). Incidental statistical summary representation over time. Journal of Vision, 16(2016), 1-14. https://doi.org/10.1167/16.3.3.doi

Piazza, E. A., Sweeny, T. D., Wessel, D., Silver, M. A., \& Whitney, D. (2013). Humans use summary statistics to perceive auditory sequences. Psychological Science, 24(8), 1389-1397. https://doi.org/10.1177/0956797612473759

Putnam-Farr, E., \& Morewedge, C. K. (2020). Which social comparisons influence happiness with unequal pay? Journal of Experimental Psychology: General. https://doi.org/10.1037/xgeoooog65

R Core Team. (2013). R: A language and environment for statistical computing. $\mathrm{R}$ Foundation for Statistical Computing. ttp://www.r-project.org/

Redelmeier, D. A., \& Kahneman, D. (1996). Patients' memories of painful medical treatments: Realtime and retrospective evaluations of two minimally invasive procedures. Pain, 66(1), 3-8. https://doi.org/10.1016/o304-3959(96)02994-6

Schirmer, A., \& Adolphs, R. (2017). Emotion perception from face, voice, and touch: Comparisons and convergence. Trends in Cognitive Science, 21(3), 216-228. https://doi.org/10.1016/j.tics.2017.01.001

Schurgin, M. W., Wixted, J. T., \& Brady, T. F. (2020). Psychophysical scaling reveals a unified theory of visual memory strength. Nature Human Behaviour, 4(11), 1156-1172. 
https://doi.org/10.1038/s41562-020-00938-o

Sessa, P., Luria, R., Gotler, A., Jolicœur, P., \& Dell'acqua, R. (2011). Interhemispheric ERP asymmetries over inferior parietal cortex reveal differential visual working memory maintenance for fearful versus neutral facial identities. Psychophysiology, 48(2), 187-197. https://doi.org/10.1111/j.14698986.2010.01046.x

Tong, K., Dubé, C., \& Sekuler, R. (2019). What makes a prototype a prototype? Averaging visual features in a sequence. Attention, Perception, and Psychophysics, 81(6), 1962-1978. https://doi.org/10.3758/s13414-019-01697-5

Tottenham, N., Tanaka, J. W., Leon, A. C., McCarry, T., Nurse, M., Hare, T. A., Marcus, D. J., Westerlund, A., Casey, B. J., \& Nelson, C. (2009). The NimStim set of facial expressions: Judgments from untrained research participants. Psychiatry Research, 168(3), 242-249. https://doi.org/10.1016/j.psychres.2008.05.006

Tsetsos, K., Chater, N., \& Usher, M. (2012). Salience driven value integration explains decision biases and preference reversal. Proceedings of the National Academy of Sciences, 109(24), 9659-9664. https://doi.org/10.1073/pnas.1119569109

Watson, D., Clark, L. A., \& Tellegen, A. (1988). Development and validation of brief measures of positive and negative affect: The PANAS scales. Journal of Personality and Social Psychology, 54(6), 1063-1070. https://doi.org/10.1037/0022-3514.54.6.1063

Whitney, D., \& Yamanashi Leib, A. (2018). Ensemble perception. Annual Review of Psychology, 69(1), 105-129. https://doi.org/10.1146/annurev-psych-010416-044232

Wickens, T. D. (2001). Elementary signal detection theory. Oxford University Press.

Wilken, P., \& Ma, W. J. (2004). A detection theory account of change detection. Journal of Vision, 4(12), 1120-1135. https://doi.org/10.1167/4.12.11

Zacks, J. M., \& Swallow, K. M. (2007). Event segmentation. Current Directions in Psychological Science, 16(2), 80-84. https://doi.org/10.1111/j.1467-8721.2007.00480.x 